\title{
Understanding Compact Object Formation and Natal Kicks I. Calculation Methods and the case of GRO J1655-40
}

\author{
B. Willems ${ }^{1}$, M. Henninger ${ }^{1}$, T. Levin ${ }^{1}$, N. Ivanova ${ }^{1}$, V. Kalogera ${ }^{1}$, K. McGhee ${ }^{2}$, F.X. \\ Timmes $^{2}$, and C.L. Fryer ${ }^{2,3}$ \\ b-willems@northwestern.edu, m-henninger@alumni.northwestern.edu, tlevin, \\ nata, vicky@northwestern.edu, kmm225@dana.ucc.nau.edu, timmes@lanl.gov, \\ fryer@lanl.gov
}

\begin{abstract}
In recent years proper motion measurements have been added to the set of observational constraints on the current properties of Galactic X-ray binaries. We develop an analysis that allows us to consider all this available information and reconstruct the full evolutionary history of X-ray binaries back to the time of core collapse and compact object formation. This analysis accounts for five evolutionary phases: mass transfer through the ongoing X-ray phase, tidal circularization before the onset of Roche-lobe overflow, motion through the Galactic potential after the formation of the compact object, binary orbital dynamics at the time of core collapse, and hydrodynamic modeling of the core collapse that connects the compact object to its progenitor and any nucleosynthetic constraints available. In this first paper, we present this analysis in a comprehensive manner and we apply it to the soft X-ray transient GRO J1655-40. This is the first analysis that incorporates all observational constraints on the current system properties and uses the full 3D peculiar velocity constraints right after core collapse instead of lower limits on the current space velocity given by the present-day radial velocity. We find that the system has remained within $200 \mathrm{pc}$ from the Galactic plane throughout its entire life time and that the mass loss and a kick possibly associated with the black hole formation imparted a kick velocity of $\simeq 45-115 \mathrm{~km} \mathrm{~s}^{-1}$ to the binary's center of mass. Right after black hole formation, the system consists of $\mathrm{a} \simeq 3.5-6.3 M_{\odot}$ black hole and $\mathrm{a} \simeq 2.3-4 M_{\odot}$ main-sequence star. At
\end{abstract}

\footnotetext{
${ }^{1}$ Northwestern University, Department of Physics and Astronomy, 2145 Sheridan Road, Evanston, IL 60208, USA

${ }^{2}$ Theoretical Division, LANL, Los Alamos, NM 87545

${ }^{3}$ Physics Dept., The University of Arizona, Tucson, AZ 85721
} 
the onset of the X-ray phase the donor is still on the main sequence. We find that a symmetric black hole formation event cannot be formally excluded, but that the associated system parameters are only marginally consistent with the currently observed binary properties. Black hole formation mechanisms involving an asymmetric supernova explosion with associated black hole kick velocities of a few tens of $\mathrm{kms}^{-1}$, on the other hand, satisfy the constraints much more comfortably. We also derive an upper limit on the black hole kick magnitude of $\simeq 210 \mathrm{~km} \mathrm{~s}^{-1}$.

Subject headings: Stars: Binaries: Close, Stars: Evolution, X-rays: Binaries, X-rays: Individual (GRO J1655-40)

\section{INTRODUCTION}

The current observed sample of Galactic black-hole (BH) X-ray binaries (XRBs) provides us with a unique opportunity for understanding the formation of black holes in binaries. In recent years the sample has grown significantly, reaching $18 \mathrm{BH}$ XRBs identified through dynamical mass measurements (Orosz 2004, McClintock \& Remillard 2004). For these systems there exists a wealth of observational information about their current physical state: $\mathrm{BH}$ and donor masses, orbital period, donor's position on the H-R diagram and surface chemical composition, transient or persistent and Roche-lobe overflow (RLO) or wind-driven character of the mass-transfer (MT) process, and distances. Even more recently proper motions have been measured for a handful of these systems (Mirabel et al. 2001, 2002; Mirabel \& Rodrigues 2003a), complementing the earlier measurements of center-of-mass radial velocities and giving us information about the 3-dimensional kinematic properties of these binaries. Similar information exists for some of the known XRBs with neutron star (NS) accretors (Ankay et al. 2001; Ribó et al. 2002; Mirabel \& Rodrigues 2003b; Mirabel, Rodrigues, \& Liu 2004).

Understanding the events of compact object formation in these known systems requires that we investigate their evolutionary history from the present state back to the time just prior to the core collapse event. In what follows we describe how the currently available observational constraints for XRBs can be used to uncover this past history and provide us with a consistent picture for how the BHs and NSs were formed. The ultimate goal of this project is to examine the systematics of the derived compact object progenitor masses and requirements for supernova $(\mathrm{SN})^{1}$ kicks and their dependence on compact object masses and

\footnotetext{
${ }^{1}$ Throughout the paper we use the term SN to describe the core-collapse event leading to the formation
} 
associated mass loss at the core collapse events. Such a comprehensive investigation will not just reveal the origin of these Galactic XRBs, but may also offer insight to the physics of supernovae and the possible association of natal kicks with $\mathrm{BH}$ formation.

Although this is not the first study that has considered the evolutionary history of known XRBs in the Galaxy (see, e.g., de Kool, van den Heuvel, \& Pylyser 1987; Brandt, Podsiadlowski, \& Sigurdsson 1995; Nelemans, Tauris, \& van den Heuvel 1999; Podsiadlowski, Rappaport, \& Pfahl 2002a; Podsiadlowski, Rappaport, \& Han 2003; Gualandris et al. 2004), it is the first one that incorporates such a large number of observational constraints for a whole sample of systems and considers the complete set of evolutionary processes from the present back to the binary stage right before compact object formation.

In this first of a series of papers, we focus on a detailed description of our analysis methodology and the rationale for combining the current constraints to complete a selfconsistent picture for the evolutionary history. We also focus on the computational methods used and the uncertainties involved, and apply our analysis to the well-studied soft X-ray transient GRO J1655-40 (Nova Sco 1994). In subsequent papers we will present our results for the rest of BH XRBs and for the NS XRBs with all the above constraints available.

The plan of the paper is as follows. In $\S 2$ we briefly review the currently available constraints on the properties of the soft X-ray transient GRO J1655-40. A general outline of the analysis used to reconstruct the system's evolutionary history is presented in $\S 3$, while the individual steps of the analysis are discussed in more detail in $\S 4-7$. In $\S 8$, we combine these steps and derive constraints on the binary's pre-SN orbital separation, on the mass of the BH's helium star progenitor, and on the kick velocity that may have been imparted to the $\mathrm{BH}$ at birth. In $\S 9$, hydrodynamic core-collapse simulations are presented and the nature of the $\mathrm{BH}$ progenitor is constrained even further. The final section is devoted to a discussion of our results and some concluding remarks.

\section{OBSERVATIONAL CONSTRAINTS FOR GRO J1655-40}

of both NSs and BHs. We are aware, however, that the occurrence of an actual SN explosion during BH formation is still an unresolved issue. The small inaccuracy in using the term SN for BHs is therefore introduced merely for brevity. In addition, for GROJ1655-40 which we consider in detail in this paper, evidence supporting a SN explosion has been presented by Israelian et al. (1999) and Podsiadlowski et al. (2002b). 
Table 1. Properties of GRO J1655-40.

\begin{tabular}{lccc}
\hline \hline \multicolumn{1}{c}{ Parameter } & Notation & & Value \\
\hline Distance & $d$ & $3.2 \pm 0.2 \mathrm{kpc}$ & (Hjellming \& Rupen 1995) \\
Galactic longitude & $l$ & $345.0^{\circ}$ & (Tingay et al. 1995) \\
Galactic latitude & $b$ & $2.2^{\circ}$ & (Tingay et al. 1995) \\
Velocity towards the Galactic Center & $U$ & $-121 \pm 18 \mathrm{~km} \mathrm{~s}^{-1}$ & (Mirabel et al. 2002) \\
Velocity in the direction of the Galactic Rotation & $V$ & $-33 \pm 8 \mathrm{~km} \mathrm{~s}^{-1}$ & (Mirabel et al. 2002) \\
Velocity towards the Northern Galactic Pole & $W$ & $3 \pm 8 \mathrm{~km} \mathrm{~s}^{-1}$ & (Mirabel et al. 2002) \\
\hline & & Greene et al. (2001) & Beer \& Podsiadlowski (2002) \\
\hline Orbital Period & $P_{\text {orb }}$ & $2.62191 \pm 0.00020$ days & $2.62168 \pm 0.00014$ days \\
Black Hole Mass & $M_{\mathrm{BH}}$ & $6.3 \pm 0.5 M_{\odot}$ & $5.40 \pm 0.30 M_{\odot}$ \\
Donor Mass & $M_{2}$ & $2.4 \pm 0.4 M_{\odot}$ & $1.45 \pm 0.35 M_{\odot}$ \\
Donor Luminosity & $L_{2}$ & $36.5 \pm 4.5 L_{\odot}$ & $21.0 \pm 6.0 L_{\odot}$ \\
Donor Effective Temperature & $T_{\text {eff } 2}$ & $6335 \pm 350 \mathrm{~K}$ & $6150 \pm 350 \mathrm{~K}$ \\
\hline
\end{tabular}

${ }^{a}$ van der Hooft et al. (1998) 
The soft X-ray transient GRO J1655-40 was first detected on 27 July 1994 by the Burst and Transient Source Experiment (BATSE) on board the Compton Gamma-Ray Observatory (Zhang et al. 1994). The optical counterpart to the X-ray source was discovered by Bailyn et al. (1995a). Shortly thereafter, Bailyn et al. (1995b) found periodic optical eclipses of unequal depth which are most likely caused by alternating eclipses of the optical component and the BH's accretion disk. The same authors also derived the orbital period to be $2.601 \pm$ 0.027 days and used the semi-amplitude of the radial-velocity variations to derive a minimum mass for the compact object of $3.16 \pm 0.15 M_{\odot}$, strongly supporting the $\mathrm{BH}$ nature of the compact star.

Since then, numerous investigations have been devoted to unraveling the properties of the BH and its companion. Spectroscopic observations led Orosz \& Bailyn (1997) to classify the optical component as an F3 IV-F6 IV star (see also Bailyn et al. 1995b), while van der Hooft et al. (1998) used photometric measurements to refine the orbital period measurement to $2.62168 \pm 0.00014$ days. The case for the BH nature of the accreting object was strengthened considerably by Soria et al. (1998) who derived a lower limit for the mass of the compact star of $5.1 M_{\odot}$, and by Phillips, Shahbaz \& Podsiadlowski (1999) and Shahbaz et al. (1999) who constrained the compact object mass to be $5.35 \pm 1.25 M_{\odot}$ and $6.7 \pm 1.2 M_{\odot}$, respectively. Evidence for the occurrence of a SN explosion was presented by Israelian et al. (1999) who reported a large overabundance of oxygen, magnesium, silicon, and sulfur in the atmosphere of the optical component. Since the latter is not massive enough to create these elements, Israelian et al. (1999) attributed the overabundances to the accretion of SN material from the BH progenitor. The distance of GRO J1655-40 from the Sun, finally, was determined by Hjellming \& Rupen (1995) using a kinematic model for the jets of the system. They derived $d=3.2 \pm 0.2 \mathrm{kpc}$, which is in excellent agreement with previous distance estimates by McKay \& Kesteven (1994), Tingay et al. (1995), and Bailyn et al. (1995a).

More recently Greene, Bailyn, \& Orosz (2001) and Beer \& Podsiadlowski (2002) have undertaken detailed studies of the ellipsoidal light variations when the system is in quiescence and derived rather different constraints for the two component masses and the position of the donor in the H-R diagram.

Greene et al. (2001) (hereafter GBO) used spherical NextGen atmosphere models for a fixed effective temperature of $6336 \mathrm{~K}$ (corresponding to a spectral type of F6 III) to model the observed B, V, I, J, K light curves without any contribution from an accretion disk surrounding the $\mathrm{BH}$. The model depends on two free parameters: the mass ratio $M_{\mathrm{BH}} / M_{2}$, where $M_{\mathrm{BH}}$ is the mass of the $\mathrm{BH}$ and $M_{2}$ the mass of the companion, and the orbital inclination $i$. As additional constraints, GBO used the radial-velocity curve derived by 
Shahbaz et al. (1999) and the projected rotational velocity $v_{\text {rot }} \sin i=93 \pm 3 \mathrm{~km} \mathrm{~s}^{-1}$ derived by Israelian et al. (1999). The authors found a BH mass $M_{\mathrm{BH}}=6.3 \pm 0.5 M_{\odot}$.

Beer \& Podsiadlowski (2002) (hereafter BP) fitted the ellipsoidal light variations in

the photometric B-, V-, R-, and I-bands using Kurucz models for the atmosphere of the Roche-lobe filling secondary and a BH accretion disk model with a flat temperature profile. Their model is characterised by five free parameters: the mass ratio $M_{\mathrm{BH}} / M_{2}$, the orbital inclination $i$, the polar temperature $T_{\text {pole }}$ of the donor star, the color excess $E(B-V)$, and the distance $d$ from the Sun. They furthermore adopted the distance of $3.2 \pm 0.2 \mathrm{kpc}$ derived by Hjellming \& Rupen (1995) as an additional ad-hoc constraint to further tighten the limits on the derived model parameters. In their best-fitting model, BP found $M_{\mathrm{BH}}=5.4 \pm 0.3 M_{\odot}$.

At present it does not seem clear which of the two analyses (GBO or BP) yields the most reliable measurements (see also Shahbaz 2003). In this study we therefore consider both sets of constraints and study them as two separate cases. An overview of all observationally inferred properties of GRO J1655-40 relevant to this investigation is presented in Table 1. In particular, these properties are the distance $d$ from the Sun, the Galactic longitude $l$ and the Galactic latitude $b$, the center-of-mass velocity components $U, V$, and $W$ with respect to a Galactic frame of reference, the orbital period $P_{\mathrm{orb}}$, the $\mathrm{BH}$ mass $M_{\mathrm{BH}}$, the donor mass $M_{2}$, the donor luminosity $L_{2}$, and the donor effective temperature $T_{\text {eff2 }}$.

\section{OUTLINE OF ANALYSIS METHODOLOGY AND BASIC ASSUMPTIONS}

According to our current understanding, the formation of a $\mathrm{BH}$ XRB with a low- to intermediate-mass donor star requires a primordial binary with an extreme mass ratio and a primary (the $\mathrm{BH}$ progenitor) mass in excess of $\simeq 20-25 M_{\odot}$. If the period of the primordial binary is in the range from $\simeq 1$ to $\simeq 10 \mathrm{yr}$, the primary is expected to become larger than its critical Roche lobe and, due to the extreme mass ratio, lose most of its hydrogen-rich envelope in a dynamically unstable common-envelope phase. Provided that enough energy is available to completely expel the envelope and avoid a merger, the common-envelope phase results in the formation of a tight binary consisting of a helium star (the core of the Rochelobe filling primary) and a relatively unevolved low- to intermediate-mass main-sequence (MS) companion. A BH XRB is then formed when the helium star collapses into a BH and stellar evolution and/or orbital angular momentum losses (or exchange through tides) cause the secondary in its turn to fill its Roche lobe and transfer mass to the BH. This formation channel is analogous to that usually considered for the formation of NS XRBs and has been considered previously by, e.g., Romani (1996), Portegies Zwart, Verbunt, \& Ergma (1997), 
Ergma \& van den Heuvel (1998), Kalogera (1999), and Podsiadlowski et al. (2003).

In this paper, we restrict ourselves to the formation of $\mathrm{BH}$ XRBs through the above "standard" evolutionary channel and do not consider alternative formation channels involving hierarchical triple star interactions (Eggleton \& Verbunt 1986) or tidal capture or exchange events in dense stellar environments (Clark 1975, Hills 1976). Hence, we assume the BH's immediate progenitor to be a helium star of mass $M_{\mathrm{He}}$ orbiting a MS companion of mass $M_{2}$. In view of the strong tidal forces operating on the binary during the commonenvelope phase of the primary, we furthermore consider the pre-SN orbit to be circular. The masses of the $\mathrm{BH}$ and its companion are assumed to remain constant until the onset of the RLO and X-ray phase.

Our goal in this analysis is to trace back the evolutionary history of known XRBs (in this paper of the soft X-ray transient GRO J1655-40) and to constrain the properties of the system's progenitor just before and right after the SN explosion that formed the $\mathrm{BH}^{2}$. The method adopted to derive the pre- and post-SN constraints incorporates the following set of calculations.

We first use a stellar evolution code and calculate a grid of evolutionary sequences for binaries in which a $\mathrm{BH}$ is accreting mass from a Roche-lobe filling companion. To consider the full range of possibilities, we include sequences for both conservative (for sub-Eddington rates) and fully non-conservative MT. For each sequence, we examine whether at any point in time the calculated binary properties, i.e., BH and donor masses, donor effective temperature and luminosity, and orbital period, are in agreement with the observational measurements or derivations of these quantities within their associated uncertainties. Among these quantities, the orbital period is measured with the highest accuracy and hence presents the most stringent constraint to be satisfied. We consider a large number of MT sequences and, although many of them can satisfy some of the constraints, the majority of sequences clearly fail to simultaneously satisfy all of the constraints at any given time. In the case of GRO J1655-40 the successful sequences furthermore have to satisfy the additional requirement that the system exhibits transient behavior. For the time interval during which all other constraints are satisfied, the long-term MT rate from the donor star must therefore be lower than than the critical rate separating transient from persistent behavior (Dubus et al. 1999; see also King, Kolb, \& Burderi 1996; King, Kolb, \& Szuszkiewicz 1997). This last requirement restricts the

\footnotetext{
${ }^{2}$ Throughout the paper we will refer to the instants just before the SN explosion and right after the formation of the $\mathrm{BH}$ by the terms pre-SN and post-SN, respectively. If the $\mathrm{BH}$ is formed via an intermediary NS stage followed by fall back of some fraction of the SN material (e.g. Brandt et al. 1995) these two times may be slightly offset from each other. We here neglect this small offset and assume the entire SN process to be instantaneous regardless of the formation mechanism of the $\mathrm{BH}$.
} 
successful sequences even further. With the remaining fully successful sequences, we derive the properties of the binary at the onset of the RLO phase: initial BH and donor masses, orbital period, and age of the donor star. The time at which the fully successful sequences satisfy all observational constraints furthermore provides an estimate for the donor's current age.

Next, we consider the kinematic evolutionary history of the XRB in the Galactic potential. In particular, we use the current position and the measured 3D velocity with their associated uncertainties to trace the Galactic motion back in time. Combined with the tight constraints on the current age of the system given by the successful MT sequences this allows us to determine the position and velocity of the binary at the time of BH formation (we denote these as the "birth" location and velocity). By subtracting the local Galactic rotational velocity at this position from the system's total center-of-mass velocity, we then obtain an estimate for the peculiar velocity of the binary right after the formation of the $\mathrm{BH}$.

The "birth" or post-SN peculiar velocity holds information about the mass loss and possibly the natal kick associated with the BH's formation. In order to extract this information we must however also constrain the orbital period and orbital eccentricity right after the formation of the $\mathrm{BH}$. We derive these constraints in the third step of our analysis: we consider pairs of post-SN orbital periods and eccentricities and integrate the equations governing the evolution of the orbit under the influence of tides and general relativity (possibly important for the most highly eccentric orbits) forward in time. By using the age of the donor star at the onset of RLO for each of the successful MT sequences as an estimate for the time expired since the formation of the $\mathrm{BH}$, we are able to map the post-SN orbital parameters to those at the onset of RLO. Comparison with the binary properties at the onset of RLO given by the sequences then allows us to select only those pairs of post-SN orbital period and eccentricity that can match these properties at the right time (i.e. at the right age or evolutionary stage of the donor star).

In the fourth step of our analysis we use the derived post-SN masses, orbital period, eccentricity, and peculiar velocity and examine the orbital dynamics of the compact object formation allowing for a natal kick. Based on angular momentum and energy conservation we derive constraints on the pre-SN binary properties (BH progenitor mass and orbital separation) and the natal kick (magnitude and direction) that may have been imparted to the $\mathrm{BH}$.

In our final step, we use the initial BH masses from the successful MT sequences and the matching range of $\mathrm{BH}$ progenitor masses to study constraints on the core collapse mechanism. By modeling the collapse of BH progenitors and the resultant SN explosion, we can calculate the final range of SN explosion energies and nucleosynthetic yields for GRO J1655-40. 
Assuming the abundance enhancements in the BH companion are due to the SN explosion that formed the $\mathrm{BH}$, we find strong evidence for the need for asymmetries in SN explosions associated with this $\mathrm{BH}$ formation event. In turn, with current stellar evolution and SN models, we can also further constrain the $\mathrm{BH}$ progenitor mass, and ultimately the pre-SN binary properties and the SN kick.

These five steps are described in more detail in the following sections.

\section{MASS TRANSFER SEQUENCES}

We calculate a set of evolutionary sequences for mass-transferring binaries using an upto-date stellar evolution code described in detail in Podsiadlowski et al. (2002a), Ivanova et al. (2003), and Kalogera et al. (2004). Binary orbits are assumed to be circular and effects of stellar rotation are neglected at all times. Mass-transfer rates are calculated self-consistently by imposing that the stellar radius remains close to the Roche-lobe radius during the entire MT phase. In order to determine whether or not a system is transient, we use the criterion

$$
\begin{aligned}
\dot{M}<\dot{M}_{\text {crit }} & \simeq 10^{-5}\left(\frac{M_{\mathrm{BH}}}{M_{\odot}}\right)^{0.5}\left(\frac{M_{2}}{M_{\odot}}\right)^{-0.2} \\
& \times\left(\frac{P_{\text {orb }}}{1 \mathrm{yr}}\right)^{1.4} M_{\odot} \mathrm{yr}^{-1}
\end{aligned}
$$

where $\dot{M}$ denotes the MT rate, and $\dot{M}_{\text {crit }}$ the critical rate separating transient from persistent behavior (Dubus et al. 1999, Kalogera et al. 2004). Mass accretion onto the BH is furthermore limited to the Eddington rate at all times and any excess mass leaving the system is assumed to carry away the specific orbital angular momentum of the accretor. Mass-loss via stellar winds is taken into account using the rates given in Hurley, Pols, \& Tout (2000). For the mixing-length and convective-overshooting parameters, finally, we adopt values of 0.2 and 0.25 pressure scale heights, respectively.

We furthermore note that all MT sequences considered have initial donor masses greater than $1.25 M_{\odot}$ so that no magnetic braking takes place in the donor star and the binary. For those sequences where MT causes the donor mass to decrease below this value by just one or two tenths of a solar mass (relevant for BP's current donor mass estimates), magnetic braking is still not included, given the relatively wide current orbit of the system.

Our goal with these calculations of MT sequences is to map out the 3-dimensional parameter space of $\mathrm{BH}$ and donor masses, and binary orbital period at the onset of RLO, for which all six observational constraints (current masses, orbital period, donor luminosity 
and effective temperature, and transient behavior) are satisfied simultaneously. Since each MT calculation takes a significant amount of computational time (from a few hours to a few days on one processor) and often requires user intervention, it is currently not feasible to develop an automated and comprehensive scheme for the systematic and exhaustive search of the entire available 3-dimensional space. Instead we therefore use a trial-and-error scheme guided strongly by the systematic behavior of the MT sequences in both the H-R diagram and the mass-period plane. We first examine the H-R tracks and adjust the RLO parameters so that tracks cross the H-R error box. Next, we identify the time intervals for which the $\mathrm{H}-\mathrm{R}$ constraints are satisfied and examine whether these parts of the tracks also cross the $\mathrm{BH}$ and donor mass errors bars and the orbital period measurement, the latter being the most stringent of all constraints. Last we examine whether the sequence exhibits transient behavior for a significant fraction of the time interval over which the other constraints are satisfied.

In this way we are able to outline the extent of the parameter space that leads to "successful" MT sequences. It turns out that this extent is small enough so that the variations among the successful sequences do not significantly affect our final conclusions for constraints on the BH XRB progenitors (see section 8), as long as we stick to a given set of observational constraints (GBO or BP). We therefore conclude that it is not necessary to strictly find all possible successful sequences, but instead to map the outline of their initial parameter space within about $0.5 \mathrm{M}_{\odot}$ in the masses and about $0.1 \mathrm{~d}$ in the orbital period.

In order to capture the uncertainties related to the accretion of matter by the $\mathrm{BH}$, we furthermore consider both conservative and non-conservative MT sequences for each of the two sets of observational constraints available for GRO J1655-40. We note that in the conservative case, mass accretion onto the $\mathrm{BH}$ is still limited to the Eddington rate and any mass transferred in excess of this rate is assumed to be lost from the system carrying the specific orbital angular momentum of the accretor. 

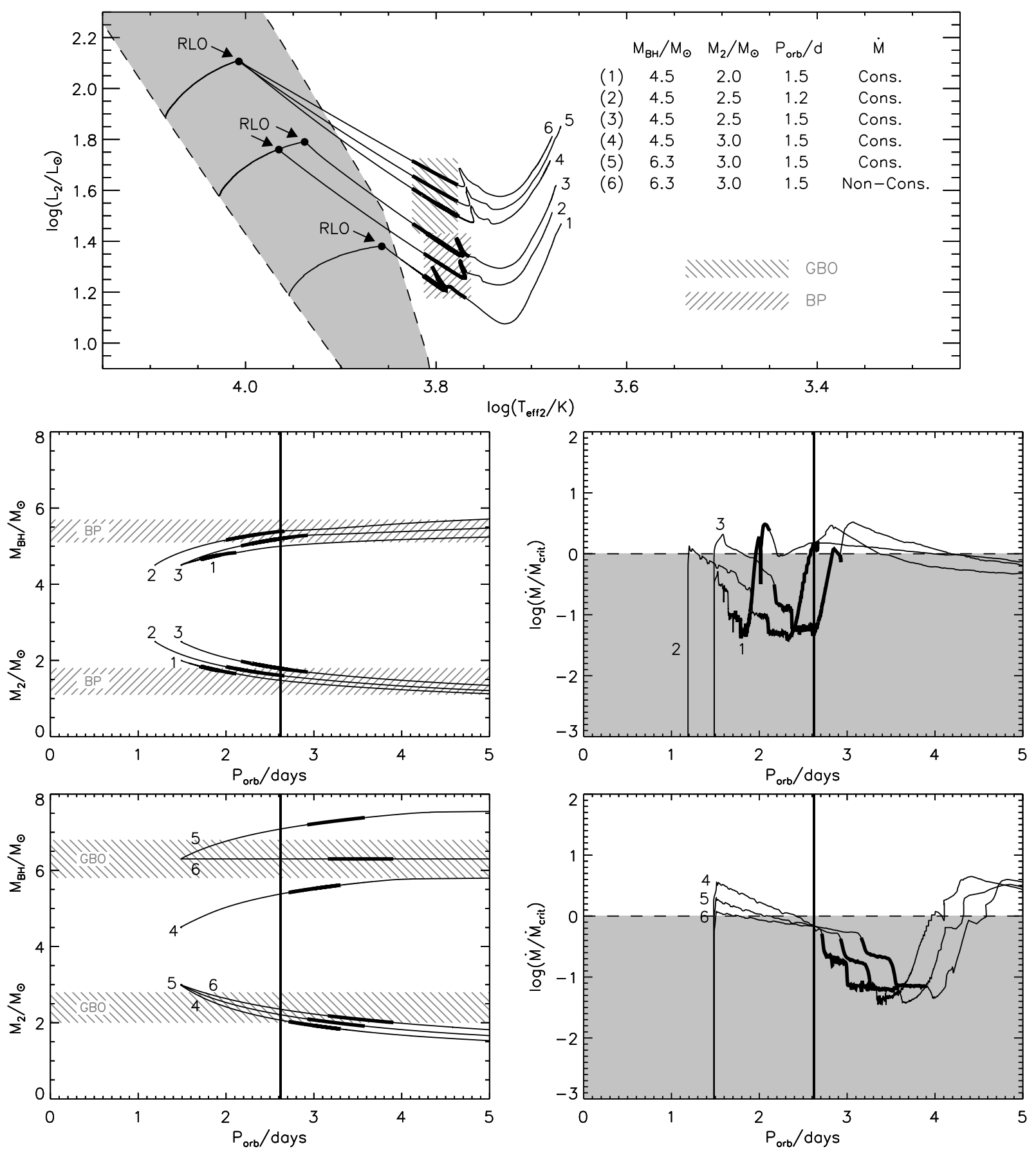

Fig. 1. - Systematic behavior of some selected MT sequences with different initial component masses and orbital periods. The top panel shows the variations of the donor star's luminosity and effective temperature on the H-R diagram. The MS for single stars is indicated by the grey-shaded region and the onset of MT by filled circles. The observational constraints derived by $\mathrm{GBO}$ and $\mathrm{BP}$ are indicated by the grey hatched regions. On the left-hand-side panels, the variations of the $\mathrm{BH}$ and donor masses are displayed as functions of the orbital period. The grey hatched regions indicate the constraints on the BH and donor mass derived by GBO and BP. On the right-hand-side panels the variations of the MT rate are displayed in units of the critical rate for transient behavior also as functions of the orbital period. Here, the grey-shaded region indicates MT rates for which the system is transient. The thick vertical line in the $\left(M, P_{\text {orb }}\right)-$ and $\left(\log \dot{M} / \dot{M}_{\text {crit }}, P_{\text {orb }}\right)$-plots indicates the observed orbital period of 2.62 days. On all five panels, the thick part of the evolutionary tracks indicates the part of the sequence where the donor star satisfies the GBO or BP constraints on the H-R diagram. 
Before describing the sequences that are able to satisfy the observational constraints for GRO J1655-40, we briefly discuss the systematic behavior of the sequences and their dependency on the initial component masses and orbital period. The systematic behavior is illustrated in Fig. 1 for some selected evolutionary tracks which were chosen mainly to provide a clear and instructive picture. The displayed sequences therefore do not represent our best possible matches to the observed properties of GRO J1655-40. For the latter, we refer to Tables 2 and 3 where we list the main properties of some selected MT sequences and where the successful sequences are indicated in boldface. Since we did not find any successful sequences with post-MS donor stars, we furthermore restricted Fig. 1 to systems with donors that are still on the MS. The initial component masses and orbital period of the sequences at the onset of MT are indicated in the top panel of the figure. Sequences 1-5 illustrate the behavior for conservative MT, while sequence 6 illustrates the behavior for non-conservative MT. For convenience, the parts of the sequences where the donor star satisfies the GBO or $\mathrm{BP}$ constraints in the H-R diagram are emphasized by thick solid lines. 
Table 2. Selected properties of MT sequences calculated to satisfy the observational constraints for GRO J1655-40 derived by GBO. The current parameters correspond to the point where the binary's orbital period is equal to the observed orbital period of 2.62 days. Successful MT sequences able to satisfy the GBO constraints as well as the transient behavior are indicated in boldface.

\begin{tabular}{|c|c|c|c|c|c|c|c|c|c|c|c|c|c|c|}
\hline \multirow[b]{2}{*}{ Sequence } & \multicolumn{5}{|c|}{ Parameters at onset of RLO } & \multicolumn{6}{|c|}{ Current parameters } & \multicolumn{3}{|c|}{ SN parameters } \\
\hline & $\begin{array}{l}M_{\mathrm{BH}}{ }^{\mathrm{a}} \\
\left(M_{\odot}\right)\end{array}$ & $\begin{array}{c}M_{2} \mathrm{~b} \\
\left(M_{\odot}\right)\end{array}$ & $\begin{array}{r}P_{\text {orb }}{ }^{\mathrm{c}} \\
\text { (days) }\end{array}$ & $X_{2}{ }^{\mathrm{d}}$ & $\begin{array}{c}\tau_{2}{ }^{\mathrm{e}} \\
(\mathrm{Myr})\end{array}$ & $\begin{array}{l}M_{\mathrm{BH}} \\
\left(M_{\odot}\right)\end{array}$ & $\begin{array}{c}M_{2} \\
\left(M_{\odot}\right)\end{array}$ & $\begin{array}{r}L_{2}{ }^{\mathrm{f}} \\
\left(L_{\odot}\right)\end{array}$ & $\begin{array}{l}T_{\text {eff } 2}{ }^{\mathrm{g}} \\
(\mathrm{K})\end{array}$ & $X_{2}$ & $\begin{array}{c}\tau_{2} \\
(\mathrm{Myr})\end{array}$ & $\begin{array}{c}V_{\text {pec,postSN }}{ }^{\mathrm{h}} \\
\left(\mathrm{km} \mathrm{s}^{-1}\right)\end{array}$ & $\begin{array}{c}M_{\mathrm{He}}{ }^{\mathrm{i}} \\
\left(M_{\odot}\right)\end{array}$ & $\begin{array}{c}V_{\mathrm{k}}^{\mathrm{j}} \\
\left(\mathrm{km} \mathrm{s}^{-1}\right)\end{array}$ \\
\hline \multicolumn{15}{|c|}{ Conservative mass-transfer sequences } \\
\hline GBO1 & 5.5 & 2.5 & 1.5 & 0.238 & 498 & 6.1 & 1.8 & 1.39 & 3.78 & 0.054 & 643 & - & - & - \\
\hline GBO2 & 5.5 & 2.5 & 1.7 & 0.192 & 526 & 6.0 & 2.0 & 1.47 & 3.80 & 0.093 & 593 & - & - & - \\
\hline GBO3 & 5.5 & 2.5 & 1.8 & 0.172 & 537 & 6.0 & 2.0 & 1.51 & 3.81 & 0.100 & 582 & $53-110$ & $5.5-9.5$ & 40-150 \\
\hline GBO4 & 5.5 & 2.5 & 1.9 & 0.152 & 547 & 5.9 & 2.1 & 1.55 & 3.81 & 0.100 & 577 & $53-110$ & $5.5-9.5$ & $40-150$ \\
\hline GBO5 & 5.5 & 2.5 & 2.0 & 0.134 & 556 & 5.9 & 2.1 & 1.59 & 3.82 & 0.097 & 574 & $53-110$ & $5.5-9.5$ & $40-150$ \\
\hline GBO6 & 5.5 & 2.5 & 2.1 & 0.118 & 564 & 5.8 & 2.2 & 1.62 & 3.83 & 0.088 & 575 & - & - & - \\
\hline GBO7 & 5.5 & 3.0 & 1.2 & 0.358 & 252 & 6.6 & 1.9 & 1.53 & 3.82 & 0.181 & 362 & - & - & - \\
\hline GBO8 & 5.5 & 3.0 & 1.3 & 0.314 & 303 & 6.5 & 2.0 & 1.59 & 3.83 & 0.179 & 392 & $51-108$ & $5.5-9.5$ & $40-160$ \\
\hline GBO9 & 5.5 & 3.0 & 1.4 & 0.284 & 318 & 6.4 & 2.1 & 1.64 & 3.84 & 0.174 & 385 & - & - & - \\
\hline GBO10 & 5.5 & 3.0 & 1.5 & 0.257 & 331 & 6.3 & 2.2 & 1.69 & 3.85 & 0.169 & 380 & - & - & - \\
\hline GBO11 & 5.5 & 3.0 & 1.6 & 0.246 & 301 & 6.3 & 2.2 & 1.73 & 3.85 & 0.165 & 333 & - & - & - \\
\hline GBO12 & 5.5 & 3.0 & 1.7 & 0.223 & 310 & 6.2 & 2.3 & 1.78 & 3.86 & 0.157 & 333 & - & - & - \\
\hline GBO13 & 5.5 & 3.0 & 1.8 & 0.203 & 317 & 6.1 & 2.4 & 1.83 & 3.87 & 0.147 & 334 & - & - & - \\
\hline GBO14 & 5.5 & 3.0 & 1.9 & 0.185 & 323 & 5.9 & 2.5 & 1.88 & 3.88 & 0.135 & 336 & - & - & - \\
\hline GBO15 & 5.5 & 3.0 & 2.0 & 0.166 & 329 & 5.8 & 2.5 & 1.92 & 3.89 & 0.124 & 339 & - & - & - \\
\hline GBO16 & 5.5 & 3.5 & 0.8 & 0.568 & 90 & 7.2 & 1.8 & 1.49 & 3.81 & 0.182 & 396 & - & - & - \\
\hline GBO17 & 5.5 & 3.5 & 1.0 & 0.456 & 148 & 7.0 & 2.0 & 1.64 & 3.84 & 0.206 & 302 & - & - & - \\
\hline GBO18 & 5.5 & 3.5 & 1.1 & 0.422 & 146 & 6.9 & 2.1 & 1.71 & 3.85 & 0.209 & 246 & - & - & - \\
\hline GBO19 & 5.5 & 3.5 & 1.2 & 0.385 & 160 & 6.8 & 2.2 & 1.77 & 3.86 & 0.207 & 234 & - & - & - \\
\hline GBO20 & 5.5 & 3.5 & 1.3 & 0.362 & 170 & 6.7 & 2.3 & 1.82 & 3.88 & 0.203 & 225 & - & - & - \\
\hline GBO21 & 5.5 & 3.5 & 1.4 & 0.320 & 180 & 6.6 & 2.3 & 1.88 & 3.89 & 0.198 & 221 & - & - & - \\
\hline GBO22 & 5.5 & 3.5 & 1.5 & 0.292 & 188 & 6.4 & 2.4 & 1.94 & 3.90 & 0.190 & 218 & - & - & - \\
\hline GBO23 & 5.5 & 3.5 & 1.6 & 0.268 & 195 & 6.2 & 2.6 & 2.00 & 3.91 & 0.180 & 217 & - & - & - \\
\hline GBO24 & 6.0 & 2.5 & 1.7 & 0.193 & 525 & 6.5 & 2.0 & 1.48 & 3.80 & 0.098 & 588 & - & - & - \\
\hline GBO25 & 6.0 & 2.5 & 1.8 & 0.243 & 526 & 6.5 & 2.0 & 1.52 & 3.81 & 0.103 & 569 & $52-105$ & $6.0-10.0$ & $40-150$ \\
\hline GBO26 & 6.0 & 2.5 & 1.9 & 0.153 & 546 & 6.4 & 2.1 & 1.56 & 3.82 & 0.101 & 576 & $52-110$ & $6.0-10.5$ & $40-150$ \\
\hline GBO27 & 6.0 & 2.5 & 2.0 & 0.127 & 555 & 6.3 & 2.1 & 1.60 & 3.82 & 0.092 & 574 & $53-110$ & $6.0-10.5$ & $40-150$ \\
\hline GBO28 & 6.0 & 2.5 & 2.1 & 0.109 & 564 & 6.3 & 2.2 & 1.63 & 3.83 & 0.085 & 576 & - & - & - \\
\hline GBO29 & 6.0 & 2.5 & 2.2 & 0.103 & 571 & 6.2 & 2.3 & 1.67 & 3.84 & 0.076 & 580 & - & - & - \\
\hline GBO30 & 6.0 & 3.0 & 1.2 & 0.350 & 284 & 7.0 & 2.0 & 1.56 & 3.82 & 0.175 & 407 & - & - & - \\
\hline
\end{tabular}


Table 2-Continued

\begin{tabular}{|c|c|c|c|c|c|c|c|c|c|c|c|c|c|c|}
\hline \multirow[b]{2}{*}{ Sequence } & \multicolumn{5}{|c|}{ Parameters at onset of RLO } & \multicolumn{6}{|c|}{ Current parameters } & \multicolumn{3}{|c|}{ SN parameters } \\
\hline & $\begin{array}{l}M_{\mathrm{BH}}{ }^{\mathrm{a}} \\
\left(M_{\odot}\right) \\
\end{array}$ & $\begin{array}{r}M_{2}{ }^{\mathrm{b}} \\
\left(M_{\odot}\right) \\
\end{array}$ & $\begin{array}{r}P_{\text {orb }}{ }^{c} \\
\text { (days) }\end{array}$ & $X_{2}{ }^{\mathrm{d}}$ & $\begin{array}{c}\tau_{2}{ }^{\mathrm{e}} \\
(\mathrm{Myr}) \\
\end{array}$ & $\begin{array}{l}M_{\mathrm{BH}} \\
\left(M_{\odot}\right)\end{array}$ & $\begin{array}{c}M_{2} \\
\left(M_{\odot}\right) \\
\end{array}$ & $\begin{array}{c}L_{2}{ }^{\mathrm{f}} \\
\left(L_{\odot}\right)\end{array}$ & $\begin{array}{c}T_{\text {eff } 2^{\mathrm{g}}} \\
(\mathrm{K})\end{array}$ & $X_{2}$ & $\begin{array}{c}\tau_{2} \\
(\mathrm{Myr})\end{array}$ & $\begin{array}{c}V_{\text {pec,postSN }}{ }^{\mathrm{h}} \\
\left(\mathrm{km} \mathrm{s}^{-1}\right)\end{array}$ & $\begin{array}{l}M_{\mathrm{He}} \mathrm{e}^{\mathrm{i}} \\
\left(M_{\odot}\right)\end{array}$ & $\begin{array}{c}V_{\mathrm{k}}^{\mathrm{j}} \\
\left(\mathrm{km} \mathrm{s}^{-1}\right)\end{array}$ \\
\hline GBO31 & 6.0 & 3.0 & 1.3 & 0.327 & 267 & 7.0 & 2.0 & 1.61 & 3.83 & 0.180 & 345 & - & - & - \\
\hline GBO32 & 6.0 & 3.0 & 1.4 & 0.287 & 280 & 6.9 & 2.1 & 1.66 & 3.84 & 0.173 & 339 & - & - & - \\
\hline GBO33 & 6.0 & 3.0 & 1.5 & 0.258 & 330 & 6.8 & 2.2 & 1.71 & 3.85 & 0.165 & 381 & - & - & - \\
\hline GBO34 & 6.0 & 3.0 & 1.6 & 0.247 & 301 & 6.7 & 2.3 & 1.75 & 3.86 & 0.161 & 335 & - & - & - \\
\hline GBO35 & 6.0 & 3.0 & 1.7 & 0.213 & 309 & 6.7 & 2.3 & 1.80 & 3.87 & 0.152 & 334 & - & - & - \\
\hline GBO36 & 6.0 & 3.0 & 1.8 & 0.190 & 358 & 6.6 & 2.4 & 1.84 & 3.88 & 0.143 & 379 & - & - & - \\
\hline GBO37 & 6.0 & 3.0 & 1.9 & 0.172 & 322 & 6.5 & 2.5 & 1.88 & 3.88 & 0.134 & 336 & - & - & - \\
\hline GBO38 & 6.0 & 3.0 & 2.0 & 0.155 & 328 & 6.4 & 2.6 & 1.93 & 3.89 & 0.123 & 338 & - & - & - \\
\hline
\end{tabular}

Non-conservative mass-transfer sequences

\begin{tabular}{|c|c|c|c|c|c|c|c|c|c|c|c|c|c|c|}
\hline GBO39 & 6.3 & 2.2 & 1.4 & 0.244 & 712 & 6.3 & 1.7 & 1.27 & 3.76 & 0.049 & 938 & - & - & - \\
\hline GBO40 & 6.3 & 2.2 & 1.5 & 0.257 & 727 & 6.3 & 1.8 & 1.31 & 3.77 & 0.044 & 913 & - & - & - \\
\hline GBO41 & 6.3 & 2.2 & 1.6 & 0.193 & 756 & 6.3 & 1.8 & 1.35 & 3.77 & 0.033 & 914 & - & - & - \\
\hline GBO42 & 6.3 & 2.2 & 1.7 & 0.171 & 774 & 6.3 & 1.8 & 1.38 & 3.78 & 0.053 & 905 & - & - & - \\
\hline GBO43 & 6.3 & 2.2 & 1.8 & 0.201 & 781 & 6.3 & 1.9 & 1.41 & 3.79 & 0.052 & 888 & - & - & - \\
\hline GBO44 & 6.3 & 2.2 & 1.9 & 0.131 & 803 & 6.3 & 1.9 & 1.45 & 3.79 & 0.004 & 893 & - & - & - \\
\hline GBO45 & 6.3 & 2.2 & 2.0 & 0.117 & 803 & 6.3 & 2.0 & 1.47 & 3.80 & 0.062 & 875 & - & - & - \\
\hline GBO46 & 6.3 & 2.2 & 2.1 & 0.142 & 818 & 6.3 & 2.0 & 1.58 & 3.83 & 0.008 & 1201 & - & - & - \\
\hline GBO47 & 6.3 & 2.2 & 2.2 & 0.135 & 831 & 6.3 & 2.0 & 1.64 & 3.84 & 0.008 & 1324 & - & - & - \\
\hline GBO48 & 6.3 & 2.6 & 1.0 & 0.455 & 319 & 6.3 & 1.8 & 1.34 & 3.77 & 0.024 & 662 & - & - & - \\
\hline GBO49 & 6.3 & 2.6 & 1.2 & 0.336 & 386 & 6.3 & 1.9 & 1.43 & 3.79 & 0.088 & 578 & - & - & - \\
\hline GBO50 & 6.3 & 2.6 & 1.3 & 0.302 & 409 & 6.3 & 1.9 & 1.47 & 3.80 & 0.111 & 546 & - & - & - \\
\hline GBO51 & 6.3 & 2.6 & 1.4 & 0.331 & 417 & 6.3 & 2.0 & 1.51 & 3.81 & 0.121 & 520 & $52-114$ & $6.5-10.5$ & $40-160$ \\
\hline GBO52 & 6.3 & 2.6 & 1.5 & 0.641 & 495 & 6.3 & 2.1 & 1.56 & 3.82 & 0.152 & 583 & $53-110$ & $6.5-10.5$ & $40-150$ \\
\hline GBO53 & 6.3 & 2.6 & 1.6 & 0.292 & 446 & 6.3 & 2.1 & 1.59 & 3.82 & 0.124 & 503 & $53-111$ & $6.5-11.0$ & $40-150$ \\
\hline GBO54 & 6.3 & 2.6 & 1.7 & 0.202 & 468 & 6.3 & 2.2 & 1.62 & 3.83 & 0.114 & 5.13 & - & - & - \\
\hline GBO55 & 6.3 & 2.6 & 1.8 & 0.169 & 540 & 6.3 & 2.3 & 1.68 & 3.84 & 0.095 & 5.82 & - & - & - \\
\hline GBO56 & 6.3 & 3.0 & 0.9 & 0.500 & 175 & 6.3 & 1.9 & 1.51 & 3.81 & 0.166 & 4.06 & - & - & - \\
\hline GBO57 & 6.3 & 3.0 & 1.0 & 0.433 & 234 & 6.3 & 2.0 & 1.57 & 3.82 & 0.166 & 429 & $53-109$ & $6.5-10.5$ & $30-160$ \\
\hline GBO58 & 6.3 & 3.0 & 1.1 & 0.406 & 231 & 6.3 & 2.1 & 1.63 & 3.83 & 0.170 & 360 & - & - & - \\
\hline GBO59 & 6.3 & 3.0 & 1.2 & 0.350 & 284 & 6.3 & 2.1 & 1.68 & 3.84 & 0.161 & 400 & - & - & - \\
\hline
\end{tabular}


Table 2-Continued

\begin{tabular}{|c|c|c|c|c|c|c|c|c|c|c|c|c|c|c|}
\hline \multirow[b]{2}{*}{ Sequence } & \multicolumn{5}{|c|}{ Parameters at onset of RLO } & \multicolumn{6}{|c|}{ Current parameters } & \multicolumn{3}{|c|}{ SN parameters } \\
\hline & $\begin{array}{l}M_{\mathrm{BH}}{ }^{\mathrm{a}} \\
\left(M_{\odot}\right)\end{array}$ & $\begin{array}{c}M_{2}^{\mathrm{b}} \\
\left(M_{\odot}\right)\end{array}$ & $\begin{array}{r}P_{\text {orb }}{ }^{c} \\
\text { (days) }\end{array}$ & $X_{2}{ }^{\mathrm{d}}$ & $\begin{array}{c}\tau_{2}{ }^{\mathrm{e}} \\
(\mathrm{Myr})\end{array}$ & $\begin{array}{l}M_{\mathrm{BH}} \\
\left(M_{\odot}\right)\end{array}$ & $\begin{array}{c}M_{2} \\
\left(M_{\odot}\right)\end{array}$ & $\begin{array}{r}L_{2}{ }^{\mathrm{f}} \\
\left(L_{\odot}\right)\end{array}$ & $\begin{array}{c}T_{\text {eff2 }}{ }^{\mathrm{g}} \\
(\mathrm{K})\end{array}$ & $X_{2}$ & $\begin{array}{c}\tau_{2} \\
(\mathrm{Myr})\end{array}$ & $\begin{array}{c}V_{\text {pec,postSN }}{ }^{\mathrm{h}} \\
\left(\mathrm{km} \mathrm{s}^{-1}\right)\end{array}$ & $\begin{array}{l}M_{\mathrm{He}}{ }^{\mathrm{i}} \\
\left(M_{\odot}\right)\end{array}$ & $\begin{array}{c}V_{\mathrm{k}}^{\mathrm{j}} \\
\left(\mathrm{km} \mathrm{s}^{-1}\right)\end{array}$ \\
\hline GBO60 & 6.3 & 3.0 & 1.3 & 0.603 & 301 & 6.3 & 2.2 & 1.73 & 3.85 & 0.186 & 392 & - & - & - \\
\hline GBO61 & 6.3 & 3.0 & 1.4 & 0.306 & 279 & 6.3 & 2.3 & 1.77 & 3.86 & 0.154 & 341 & - & - & - \\
\hline GBO62 & 6.3 & 3.0 & 1.5 & 0.271 & 291 & 6.3 & 2.4 & 1.81 & 3.87 & 0.148 & 341 & - & - & - \\
\hline GBO63 & 6.3 & 3.3 & 0.7 & 0.637 & 52 & 6.3 & 1.9 & 1.51 & 3.81 & 0.171 & 383 & - & - & - \\
\hline GBO64 & 6.3 & 3.3 & 0.8 & 0.569 & 96 & 6.3 & 2.0 & 1.60 & 3.83 & 0.186 & 332 & - & - & - \\
\hline GBO65 & 6.3 & 3.3 & 0.9 & 0.501 & 152 & 6.3 & 2.2 & 1.73 & 3.86 & 0.171 & 354 & - & - & - \\
\hline GBO66 & 6.3 & 3.3 & 1.0 & 0.459 & 154 & 6.3 & 2.2 & 1.73 & 3.86 & 0.185 & 289 & - & - & - \\
\hline GBO67 & 6.3 & 3.3 & 1.1 & 0.416 & 173 & 6.3 & 2.3 & 1.79 & 3.87 & 0.181 & 278 & - & - & - \\
\hline
\end{tabular}

${ }^{\mathrm{a}} \mathrm{BH}$ mass

${ }^{\mathrm{b}}$ Donor mass

${ }^{\mathrm{c}}$ Orbital period

${ }^{\mathrm{d}}$ Central hydrogen fraction

eAge

${ }^{\mathrm{f}}$ Donor luminosity

${ }^{g}$ Donor effective temperature

${ }^{\mathrm{h}}$ Post-SN peculiar velocity

'Mass of the BH's helium star progenitor

${ }^{\mathrm{j}}$ Kick velocity imparted to the $\mathrm{BH}$ 
Table 3. Selected properties of mass-transfer sequences calculated to satisfy the observational constraints for GRO J1655-40 derived by BP. Symbols have the same meaning as in Table 2.

\begin{tabular}{|c|c|c|c|c|c|c|c|c|c|c|c|c|c|c|}
\hline \multirow[b]{2}{*}{ Sequence } & \multicolumn{5}{|c|}{ Parameters at onset of RLO } & \multicolumn{6}{|c|}{ Current parameters } & \multicolumn{3}{|c|}{ SN parameters } \\
\hline & $\begin{array}{l}M_{\mathrm{BH}} \\
\left(M_{\odot}\right)\end{array}$ & $\begin{array}{c}M_{2} \\
\left(M_{\odot}\right)\end{array}$ & $\begin{array}{c}P_{\text {orb }} \\
\text { (days) }\end{array}$ & $X_{2}$ & $\begin{array}{c}\tau_{2} \\
(\mathrm{Myr})\end{array}$ & $\begin{array}{l}M_{\mathrm{BH}} \\
\left(M_{\odot}\right)\end{array}$ & $\begin{array}{c}M_{2} \\
\left(M_{\odot}\right)\end{array}$ & $\begin{array}{c}L_{2} \\
\left(L_{\odot}\right)\end{array}$ & $\begin{array}{c}T_{\text {eff2 }} \\
(\mathrm{K})\end{array}$ & $X_{2}$ & $\begin{array}{c}\tau_{2} \\
(\mathrm{Myr})\end{array}$ & $\begin{array}{c}V_{\text {pec,postSN }} \\
\left(\mathrm{km} \mathrm{s}^{-1}\right)\end{array}$ & $\begin{array}{c}M_{\mathrm{He}} \\
\left(M_{\odot}\right)\end{array}$ & $\begin{array}{c}V_{\mathrm{k}} \\
\left(\mathrm{km} \mathrm{s}^{-1}\right)\end{array}$ \\
\hline \multicolumn{15}{|c|}{ Conservative mass-transfer sequences } \\
\hline BP1 & 3.5 & 3.0 & 0.6 & 0.682 & 18 & 5.3 & 1.2 & 1.04 & 3.73 & 0.090 & 1078 & - & - & - \\
\hline BP2 & 3.5 & 3.0 & 0.7 & 0.599 & 94 & 5.2 & 1.3 & 1.13 & 3.74 & 0.052 & 902 & - & - & - \\
\hline BP3 & 3.5 & 3.0 & 0.8 & 0.541 & 147 & 5.1 & 1.4 & 1.19 & 3.75 & 0.036 & 782 & - & - & - \\
\hline $\mathrm{BP} 4$ & 3.5 & 3.0 & 0.9 & 0.485 & 185 & 5.0 & 1.5 & 1.26 & 3.77 & 0.003 & 700 & - & - & - \\
\hline BP5 & 3.5 & 3.0 & 1.0 & 0.429 & 215 & 4.9 & 1.5 & 1.26 & 3.76 & 0.031 & 619 & - & - & - \\
\hline BP6 & 3.5 & 3.5 & 1.1 & 0.409 & 151 & 4.9 & 1.8 & 1.49 & 3.81 & 0.216 & 278 & - & - & - \\
\hline BP7 & 3.5 & 3.0 & 1.2 & 0.348 & 257 & 4.7 & 1.7 & 1.36 & 3.78 & 0.130 & 446 & - & - & - \\
\hline BP8 & 3.5 & 3.5 & 0.6 & 0.698 & 1 & 5.6 & 1.3 & 1.15 & 3.75 & 0.056 & 885 & - & - & - \\
\hline BP9 & 3.5 & 3.5 & 0.7 & 0.627 & 51 & 5.5 & 1.4 & 1.28 & 3.77 & 0.004 & 730 & $47-112$ & $4.0-6.0$ & $40-200$ \\
\hline BP10 & 3.5 & 3.5 & 0.8 & 0.569 & 87 & 5.4 & 1.5 & 1.28 & 3.77 & 0.059 & 580 & $52-110$ & $3.5-6.0$ & $30-200$ \\
\hline BP11 & 3.5 & 3.5 & 0.9 & 0.511 & 114 & 5.3 & 1.6 & 1.35 & 3.78 & 0.128 & 436 & 49-109 & $3.5-6.0$ & $0-210$ \\
\hline BP12 & 3.5 & 3.5 & 1.0 & 0.463 & 134 & 5.1 & 1.7 & 1.42 & 3.80 & 0.184 & 335 & $52-111$ & $3.5-6.5$ & $0-210$ \\
\hline BP13 & 3.5 & 3.5 & 1.1 & 0.409 & 151 & 4.9 & 1.8 & 1.49 & 3.81 & 0.216 & 278 & - & - & - \\
\hline BP14 & 3.5 & 3.5 & 1.2 & 0.371 & 164 & 4.7 & 1.9 & 1.56 & 3.82 & 0.229 & 241 & - & - & - \\
\hline $\mathrm{BP} 15$ & 3.5 & 4.0 & 0.6 & 0.699 & 1 & 5.8 & 1.4 & 1.27 & 3.76 & 0.052 & 764 & - & - & - \\
\hline BP16 & 3.5 & 4.0 & 0.7 & 0.639 & 28 & 5.8 & 1.5 & 1.30 & 3.77 & 0.049 & 580 & - & - & - \\
\hline BP17 & 3.5 & 4.0 & 0.8 & 0.583 & 55 & 5.5 & 1.6 & 1.41 & 3.80 & 0.157 & 386 & $55-106$ & $4.0-6.0$ & $30-210$ \\
\hline BP18 & 3.5 & 4.0 & 0.9 & 0.515 & 74 & 5.4 & 1.7 & 1.49 & 3.81 & 0.199 & 290 & - & - & - \\
\hline BP19 & 3.5 & 4.0 & 1.0 & 0.461 & 91 & 5.2 & 1.8 & 1.58 & 3.83 & 0.223 & 234 & - & - & - \\
\hline BP20 & 3.5 & 4.0 & 1.1 & 0.419 & 102 & 4.9 & 1.9 & 1.66 & 3.85 & 0.236 & 196 & - & - & - \\
\hline BP21 & 3.5 & 4.5 & 0.7 & 0.654 & 16 & 5.9 & 1.6 & 1.38 & 3.79 & 0.105 & 461 & - & - & - \\
\hline $\mathrm{BP} 22$ & 3.5 & 4.5 & 0.8 & 0.588 & 37 & 5.7 & 1.7 & 1.50 & 3.82 & 0.171 & 317 & - & - & - \\
\hline BP23 & 3.5 & 4.5 & 0.9 & 0.529 & 53 & 5.5 & 1.8 & 1.61 & 3.84 & 0.199 & 245 & - & - & - \\
\hline BP24 & 3.5 & 4.5 & 1.0 & 0.476 & 65 & 5.2 & 1.9 & 1.70 & 3.86 & 0.218 & 196 & - & - & - \\
\hline $\mathrm{BP} 25$ & 4.0 & 2.5 & 0.6 & 0.661 & 64 & 5.4 & 1.1 & 0.97 & 3.71 & 0.121 & 1.320 & - & - & - \\
\hline BP26 & 4.0 & 2.5 & 0.7 & 0.575 & 186 & 5.3 & 1.2 & 1.02 & 3.72 & 0.100 & 1.133 & - & - & - \\
\hline $\mathrm{BP} 27$ & 4.0 & 2.5 & 0.8 & 0.504 & 268 & 5.2 & 1.3 & 1.07 & 3.73 & 0.071 & 1.004 & - & - & - \\
\hline BP28 & 4.0 & 2.5 & 0.9 & 0.486 & 323 & 5.1 & 1.4 & 1.12 & 3.74 & 0.048 & 908 & - & - & - \\
\hline BP29 & 4.0 & 2.5 & 1.0 & 0.441 & 367 & 5.0 & 1.4 & 1.15 & 3.74 & 0.038 & 844 & - & - & - \\
\hline BP30 & 4.0 & 2.5 & 1.1 & 0.358 & 411 & 5.0 & 1.5 & 1.19 & 3.75 & 0.000 & 806 & - & - & - \\
\hline
\end{tabular}


Table 3-Continued

\begin{tabular}{|c|c|c|c|c|c|c|c|c|c|c|c|c|c|c|}
\hline \multirow[b]{2}{*}{ Sequence } & \multicolumn{5}{|c|}{ Parameters at onset of RLO } & \multicolumn{6}{|c|}{ Current parameters } & \multicolumn{3}{|c|}{ SN parameters } \\
\hline & $\begin{array}{l}M_{\mathrm{BH}} \\
\left(M_{\odot}\right)\end{array}$ & $\begin{array}{c}M_{2} \\
\left(M_{\odot}\right)\end{array}$ & $\begin{array}{c}P_{\text {orb }} \\
\text { (days) }\end{array}$ & $X_{2}$ & $\begin{array}{c}\tau_{2} \\
(\mathrm{Myr})\end{array}$ & $\begin{array}{l}M_{\mathrm{BH}} \\
\left(M_{\odot}\right)\end{array}$ & $\begin{array}{c}M_{2} \\
\left(M_{\odot}\right)\end{array}$ & $\begin{array}{c}L_{2} \\
\left(L_{\odot}\right)\end{array}$ & $\begin{array}{c}T_{\text {eff2 }} \\
(\mathrm{K})\end{array}$ & $X_{2}$ & $\begin{array}{c}\tau_{2} \\
(\mathrm{Myr})\end{array}$ & $\begin{array}{c}V_{\text {pec,postSN }} \\
\left(\mathrm{km} \mathrm{s}^{-1}\right)\end{array}$ & $\begin{array}{c}M_{\mathrm{He}} \\
\left(M_{\odot}\right)\end{array}$ & $\begin{array}{c}V_{\mathrm{k}} \\
\left(\mathrm{km} \mathrm{s}^{-1}\right)\end{array}$ \\
\hline BP31 & 4.0 & 2.5 & 1.2 & 0.317 & 437 & 4.9 & 1.5 & 1.24 & 3.76 & 0.046 & 768 & - & - & - \\
\hline BP32 & 4.0 & 2.5 & 1.3 & 0.288 & 464 & 4.9 & 1.6 & 1.32 & 3.77 & 0.004 & 739 & - & - & - \\
\hline BP33 & 4.0 & 3.0 & 0.6 & 0.688 & 12 & 5.7 & 1.3 & 1.09 & 3.73 & 0.063 & 989 & - & - & - \\
\hline BP34 & 4.0 & 3.0 & 0.7 & 0.604 & 90 & 5.6 & 1.4 & 1.17 & 3.75 & 0.049 & 836 & - & - & - \\
\hline BP35 & 4.0 & 3.0 & 0.8 & 0.535 & 144 & 5.5 & 1.5 & 1.27 & 3.77 & 0.001 & 733 & $51-112$ & $4.0-7.0$ & $30-190$ \\
\hline BP36 & 4.0 & 3.0 & 0.9 & 0.489 & 182 & 5.5 & 1.5 & 1.26 & 3.76 & 0.016 & 648 & $49-115$ & $4.0-7.0$ & $0-200$ \\
\hline BP37 & 4.0 & 3.0 & 1.0 & 0.450 & 244 & 5.4 & 1.6 & 1.31 & 3.77 & 0.091 & 618 & $48-115$ & $4.0-7.0$ & $0-200$ \\
\hline BP38 & 4.0 & 3.0 & 1.1 & 0.398 & 235 & 5.3 & 1.7 & 1.36 & 3.78 & 0.117 & 464 & $51-110$ & $4.0-7.0$ & 20-190 \\
\hline BP39 & 4.0 & 3.0 & 1.2 & 0.351 & 255 & 5.2 & 1.8 & 1.42 & 3.79 & 0.156 & 407 & $55-113$ & $4.0-7.5$ & $30-200$ \\
\hline BP40 & 4.0 & 3.0 & 1.3 & 0.309 & 270 & 5.1 & 1.8 & 1.47 & 3.80 & 0.176 & 365 & - & - & - \\
\hline BP41 & 4.0 & 3.0 & 1.4 & 0.279 & 283 & 5.0 & 1.9 & 1.53 & 3.81 & 0.185 & 343 & - & - & - \\
\hline BP42 & 4.0 & 3.0 & 1.5 & 0.251 & 333 & 4.9 & 2.0 & 1.59 & 3.82 & 0.185 & 375 & - & - & - \\
\hline BP43 & 4.0 & 3.5 & 0.6 & 0.699 & 1 & 6.0 & 1.4 & 1.24 & 3.75 & 0.058 & 791 & - & - & - \\
\hline BP44 & 4.0 & 3.5 & 0.7 & 0.626 & 47 & 6.0 & 1.5 & 1.27 & 3.77 & 0.016 & 651 & - & - & - \\
\hline BP45 & 4.0 & 3.5 & 0.8 & 0.566 & 85 & 5.9 & 1.6 & 1.34 & 3.78 & 0.111 & 480 & - & - & - \\
\hline $\mathrm{BP} 46$ & 4.0 & 3.5 & 0.9 & 0.499 & 111 & 5.8 & 1.7 & 1.42 & 3.80 & 0.170 & 366 & - & - & - \\
\hline BP47 & 4.0 & 3.5 & 1.0 & 0.468 & 132 & 5.6 & 1.8 & 1.49 & 3.81 & 0.208 & 298 & - & - & - \\
\hline BP48 & 4.0 & 3.5 & 1.1 & 0.401 & 149 & 5.5 & 1.9 & 1.56 & 3.82 & 0.218 & 260 & - & - & - \\
\hline BP49 & 4.0 & 3.5 & 1.2 & 0.375 & 163 & 5.3 & 2.0 & 1.63 & 3.84 & 0.223 & 238 & - & - & - \\
\hline BP50 & 4.0 & 3.5 & 1.3 & 0.341 & 174 & 5.1 & 2.1 & 1.70 & 3.85 & 0.220 & 224 & - & - & - \\
\hline BP51 & 4.5 & 2.0 & 1.2 & 0.280 & 880 & 5.2 & 1.3 & 1.03 & 3.72 & 0.094 & 1271 & - & - & - \\
\hline BP52 & 4.5 & 2.0 & 1.3 & 0.245 & 915 & 5.1 & 1.4 & 1.05 & 3.72 & 0.097 & 1277 & - & - & - \\
\hline BP53 & 4.5 & 2.0 & 1.4 & 0.217 & 959 & 5.1 & 1.4 & 1.06 & 3.72 & 0.091 & 1219 & - & - & - \\
\hline BP54 & 4.5 & 2.0 & 1.5 & 0.224 & 982 & 5.0 & 1.5 & 1.08 & 3.72 & 0.088 & 1194 & - & - & - \\
\hline BP55 & 4.5 & 2.0 & 1.6 & 0.164 & 1016 & 4.9 & 1.5 & 1.10 & 3.72 & 0.085 & 1188 & - & - & - \\
\hline BP56 & 4.5 & 2.0 & 1.7 & 0.138 & 1.032 & 4.8 & 1.6 & 1.33 & 3.76 & 0.062 & 1193 & - & - & - \\
\hline BP57 & 4.5 & 2.0 & 1.8 & 0.114 & 1.054 & 4.8 & 1.6 & 1.14 & 3.73 & 0.083 & 1752 & - & - & - \\
\hline BP58 & 4.5 & 2.0 & 1.9 & 0.091 & 1.075 & 4.8 & 1.7 & 1.17 & 3.73 & 0.078 & 2376 & - & - & - \\
\hline BP59 & 4.5 & 2.5 & 1.1 & 0.361 & 409 & 5.4 & 1.5 & 1.22 & 3.75 & 0.000 & 790 & - & - & - \\
\hline BP60 & 4.5 & 2.5 & 1.2 & 0.323 & 438 & 5.4 & 1.6 & 1.32 & 3.77 & 0.001 & 755 & - & - & - \\
\hline BP61 & 4.5 & 2.5 & 1.3 & 0.341 & 456 & 5.3 & 1.7 & 1.32 & 3.77 & 0.003 & 715 & $47-115$ & $4.5-7.5$ & $40-170$ \\
\hline BP62 & 4.5 & 2.5 & 1.4 & 0.315 & 476 & 5.3 & 1.7 & 1.33 & 3.77 & 0.009 & 689 & $52-110$ & $4.5-8.0$ & $40-170$ \\
\hline
\end{tabular}


Table 3-Continued

\begin{tabular}{|c|c|c|c|c|c|c|c|c|c|c|c|c|c|c|}
\hline \multirow[b]{2}{*}{ Sequence } & \multicolumn{5}{|c|}{ Parameters at onset of RLO } & \multicolumn{6}{|c|}{ Current parameters } & \multicolumn{3}{|c|}{ SN parameters } \\
\hline & $\begin{array}{l}M_{\mathrm{BH}} \\
\left(M_{\odot}\right)\end{array}$ & $\begin{array}{c}M_{2} \\
\left(M_{\odot}\right)\end{array}$ & $\begin{array}{c}P_{\text {orb }} \\
\text { (days) }\end{array}$ & $X_{2}$ & $\begin{array}{c}\tau_{2} \\
(\mathrm{Myr})\end{array}$ & $\begin{array}{l}M_{\mathrm{BH}} \\
\left(M_{\odot}\right)\end{array}$ & $\begin{array}{c}M_{2} \\
\left(M_{\odot}\right)\end{array}$ & $\begin{array}{c}L_{2} \\
\left(L_{\odot}\right)\end{array}$ & $\begin{array}{c}T_{\text {eff } 2} \\
(\mathrm{~K})\end{array}$ & $X_{2}$ & $\begin{array}{c}\tau_{2} \\
(\mathrm{Myr})\end{array}$ & $\begin{array}{c}V_{\text {pec,postSN }} \\
\left(\mathrm{km} \mathrm{s}^{-1}\right)\end{array}$ & $\begin{array}{l}M_{\mathrm{He}} \\
\left(M_{\odot}\right)\end{array}$ & $\begin{array}{c}V_{\mathrm{k}} \\
\left(\mathrm{km} \mathrm{s}^{-1}\right)\end{array}$ \\
\hline BP63 & 4.5 & 2.5 & 1.5 & 0.295 & 491 & 5.2 & 1.8 & 1.34 & 3.77 & 0.029 & 657 & $50-114$ & $4.5-8.0$ & $40-170$ \\
\hline BP64 & 4.5 & 2.5 & 1.6 & 0.273 & 506 & 5.1 & 1.8 & 1.39 & 3.78 & 0.053 & 623 & - & - & - \\
\hline BP65 & 4.5 & 3.0 & 0.8 & 0.544 & 141 & 6.0 & 1.5 & 1.27 & 3.77 & 0.008 & 695 & - & - & - \\
\hline BP66 & 4.5 & 3.0 & 0.9 & 0.479 & 180 & 5.9 & 1.6 & 1.29 & 3.77 & 0.046 & 593 & - & - & - \\
\hline BP67 & 4.5 & 3.0 & 1.0 & 0.436 & 211 & 5.8 & 1.7 & 1.35 & 3.78 & 0.106 & 496 & - & - & - \\
\hline BP68 & 4.5 & 3.0 & 1.1 & 0.392 & 235 & 5.7 & 1.8 & 1.41 & 3.79 & 0.145 & 429 & - & - & - \\
\hline BP69 & 4.5 & 3.0 & 1.2 & 0.354 & 254 & 5.7 & 1.8 & 1.46 & 3.80 & 0.175 & 380 & - & - & - \\
\hline BP70 & 4.5 & 3.0 & 1.3 & 0.322 & 269 & 5.6 & 1.9 & 1.52 & 3.81 & 0.184 & 356 & - & - & - \\
\hline BP71 & 4.5 & 3.0 & 1.4 & 0.292 & 282 & 5.5 & 2.0 & 1.57 & 3.82 & 0.188 & 339 & - & - & - \\
\hline BP72 & 4.5 & 3.0 & 1.5 & 0.276 & 292 & 5.4 & 2.1 & 1.63 & 3.83 & 0.182 & 331 & - & - & - \\
\hline
\end{tabular}

Non-conservative mass-transfer sequences

\begin{tabular}{|c|c|c|c|c|c|c|c|c|c|c|c|c|c|c|}
\hline BP73 & 5.4 & 2.0 & 1.0 & 0.361 & 760 & 5.4 & 1.4 & 1.03 & 3.72 & 0.099 & 1315 & - & - & - \\
\hline BP74 & 5.4 & 2.0 & 1.1 & 0.341 & 821 & 5.4 & 1.4 & 1.05 & 3.72 & 0.095 & 1267 & - & - & - \\
\hline BP75 & 5.4 & 2.0 & 1.2 & 0.304 & 872 & 5.4 & 1.5 & 1.07 & 3.72 & 0.092 & 1236 & - & - & - \\
\hline BP76 & 5.4 & 2.0 & 1.3 & 0.275 & 915 & 5.4 & 1.5 & 1.09 & 3.72 & 0.086 & 1215 & - & - & - \\
\hline BP77 & 5.4 & 2.0 & 1.4 & 0.213 & 1079 & 5.4 & 1.6 & 1.12 & 3.72 & 0.081 & 1357 & - & - & - \\
\hline BP78 & 5.4 & 2.0 & 1.5 & 0.208 & 1138 & 5.4 & 1.6 & 1.17 & 3.73 & 0.062 & 1411 & - & - & - \\
\hline BP79 & 5.4 & 2.3 & 0.8 & 0.498 & 349 & 5.4 & 1.4 & 1.10 & 3.73 & 0.066 & 1044 & - & - & - \\
\hline BP80 & 5.4 & 2.3 & 0.9 & 0.440 & 424 & 5.4 & 1.5 & 1.13 & 3.73 & 0.054 & 978 & - & - & - \\
\hline BP81 & 5.4 & 2.3 & 1.0 & 0.383 & 547 & 5.4 & 1.6 & 1.25 & 3.76 & 0.000 & 1035 & - & - & - \\
\hline BP82 & 5.4 & 2.3 & 1.1 & 0.341 & 596 & 5.4 & 1.7 & 1.29 & 3.76 & 0.000 & 998 & $46-111$ & $5.5-9.0$ & $40-160$ \\
\hline BP83 & 5.4 & 2.3 & 1.2 & 0.348 & 557 & 5.4 & 1.7 & 1.25 & 3.76 & 0.091 & 860 & - & - & - \\
\hline BP84 & 5.4 & 2.5 & 0.8 & 0.543 & 257 & 5.4 & 1.5 & 1.20 & 3.75 & 0.042 & 854 & - & - & - \\
\hline BP85 & 5.4 & 2.5 & 0.9 & 0.445 & 389 & 5.4 & 1.6 & 1.33 & 3.77 & 0.000 & 924 & 54-115 & $5.5-9.0$ & $40-170$ \\
\hline BP86 & 5.4 & 2.5 & 1.0 & 0.396 & 445 & 5.4 & 1.7 & 1.40 & 3.79 & 0.000 & 882 & $55-108$ & $5.5-8.5$ & $50-150$ \\
\hline BP87 & 5.4 & 2.5 & 1.1 & 0.402 & 399 & 5.4 & 1.7 & 1.35 & 3.78 & 0.004 & 719 & 47-115 & $5.5-9.0$ & $40-160$ \\
\hline BP88 & 5.4 & 2.5 & 1.2 & 0.326 & 436 & 5.4 & 1.8 & 1.36 & 3.78 & 0.022 & 696 & $53-110$ & $5.5-9.0$ & $40-160$ \\
\hline BP89 & 5.4 & 2.5 & 1.3 & 0.293 & 461 & 5.4 & 1.9 & 1.39 & 3.78 & 0.045 & 660 & - & - & - \\
\hline ВР90 & 5.4 & 2.5 & 1.4 & 0.265 & 468 & 5.4 & 1.9 & 1.43 & 3.79 & 0.067 & 618 & - & - & - \\
\hline BP91 & 5.4 & 3.0 & 0.6 & 0.699 & 5 & 5.4 & 1.6 & 1.33 & 3.78 & 0.004 & 841 & - & - & - \\
\hline
\end{tabular}


Table 3-Continued

\begin{tabular}{|c|c|c|c|c|c|c|c|c|c|c|c|c|c|c|}
\hline \multirow[b]{2}{*}{ Sequence } & \multicolumn{5}{|c|}{ Parameters at onset of RLO } & \multicolumn{6}{|c|}{ Current parameters } & \multicolumn{3}{|c|}{ SN parameters } \\
\hline & $\begin{array}{l}M_{\mathrm{BH}} \\
\left(M_{\odot}\right)\end{array}$ & $\begin{array}{c}M_{2} \\
\left(M_{\odot}\right) \\
\end{array}$ & $\begin{array}{c}P_{\text {orb }} \\
\text { (days) }\end{array}$ & $X_{2}$ & $\begin{array}{c}\tau_{2} \\
(\mathrm{Myr})\end{array}$ & $\begin{array}{l}M_{\mathrm{BH}} \\
\left(M_{\odot}\right)\end{array}$ & $\begin{array}{c}M_{2} \\
\left(M_{\odot}\right)\end{array}$ & $\begin{array}{c}L_{2} \\
\left(L_{\odot}\right)\end{array}$ & $\begin{array}{c}T_{\text {eff } 2} \\
(\mathrm{~K})\end{array}$ & $X_{2}$ & $\begin{array}{c}\tau_{2} \\
(\mathrm{Myr})\end{array}$ & $\begin{array}{c}V_{\text {pec,postSN }} \\
\left(\mathrm{km} \mathrm{s}^{-1}\right)\end{array}$ & $\begin{array}{c}M_{\mathrm{He}} \\
\left(M_{\odot}\right)\end{array}$ & $\begin{array}{c}V_{\mathrm{k}} \\
\left(\mathrm{km} \mathrm{s}^{-1}\right)\end{array}$ \\
\hline BP92 & 5.4 & 3.0 & 0.7 & 0.612 & 96 & 5.4 & 1.7 & 1.33 & 3.77 & 0.058 & 679 & $51-108$ & $5.5-8.5$ & $40-170$ \\
\hline BP93 & 5.4 & 3.0 & 0.8 & 0.548 & 138 & 5.4 & 1.8 & 1.41 & 3.79 & 0.120 & 485 & $52-106$ & $5.5-8.5$ & $40-160$ \\
\hline BP94 & 5.4 & 3.0 & 0.9 & 0.490 & 178 & 5.4 & 1.9 & 1.48 & 3.80 & 0.158 & 417 & - & - & - \\
\hline BP95 & 5.4 & 3.3 & 0.6 & 0.700 & 1 & 5.4 & 1.7 & 1.37 & 3.78 & 0.089 & 531 & - & - & - \\
\hline BP96 & 5.4 & 3.3 & 0.7 & 0.633 & 55 & 5.4 & 1.8 & 1.47 & 3.80 & 0.163 & 400 & - & - & - \\
\hline BP97 & 5.4 & 3.3 & 0.8 & 0.566 & 98 & 5.4 & 1.9 & 1.56 & 3.82 & 0.187 & 340 & - & - & - \\
\hline BP98 & 5.4 & 3.3 & 0.9 & 0.507 & 131 & 5.4 & 2.0 & 1.63 & 3.84 & 0.190 & 309 & - & - & - \\
\hline
\end{tabular}


The shape of the evolutionary tracks in the H-R diagram is similar for all considered sequences: the donor star follows its normal MS evolution until the onset of MT (indicated by the filled circles) and then evolves towards lower luminosities and lower effective temperatures as it adjusts to its decreasing mass. The evolutionary stage of the donor at the onset of MT is determined by the the orbital period at the start of the RLO phase. For a given $\mathrm{BH}$ and donor mass, longer initial periods yield more evolved donor stars and higher initial MT rates. Since tracks with longer initial periods are also associated with larger donor stars, they furthermore tend to be shifted towards higher luminosities (cf. sequences 2 and 3). A similar tendency occurs when increasing the donor mass for a fixed BH mass and initial orbital period (cf. sequences 1, 3, and 4). The requirement that the sequences must pass through the observationally inferred position of GRO J1655-40 in the H-R diagram therefore gives a rough lower and upper limit on the initial period and donor mass at the onset of RLO.

For a given donor mass and a given initial period, the BH mass and the amount of mass lost from the system during the MT process affect the evolution in the H-R diagram through the rate at which the donor reduces its mass. Higher BH masses and non-conservative MT yield lower MT rates and therefore a slower decrease of the donor's luminosity and effective temperature during the MT phase compared to that for lower BH masses and conservative MT (cf. sequences 4, 5, and 6). These effects are less severe though than changes in the initial donor mass and orbital period.

The variations of the MT rate expressed in units of the critical rate for transient behavior depend on the relative changes between these two quantities. After the onset of the MT phase, the decrease of the mass ratio and the increase of the orbital period cause the MT rate to decrease and the critical rate to increase, so that overall the ratio $\dot{M} / \dot{M}_{\text {crit }}$ decreases with increasing orbital period. This tendency continues until the donor reaches the end of core-hydrogen burning and the associated expansion of the stellar radius causes the MT rate to increase more rapidly than the critical rate for transient behavior. The ratio $\dot{M} / \dot{M}_{\text {crit }}$ therefore increases as well. During the subsequent evolution through the Hertzsprung gap, no nuclear burning takes place and the MT rate stays roughly constant. Since $\dot{M}_{\text {crit }}$ still increases slowly, the ratio $\dot{M} / \dot{M}_{\text {crit }}$ slowly decreases.

Examination of Fig. 1 indicates how we can effectively identify winner sequences. All six sequences shown as an example satisfy the GBO or BP constraints in the H-R diagram, but only two of them (sequence 2 and 3) satisfy the BP mass constraints, and only one of those satisfies the transient constraint (sequence 3). The MT sequences which are able to satisfy the constraints derived by GBO and BP are highlighted in boldface in Tables 2 and 3, respectively. 


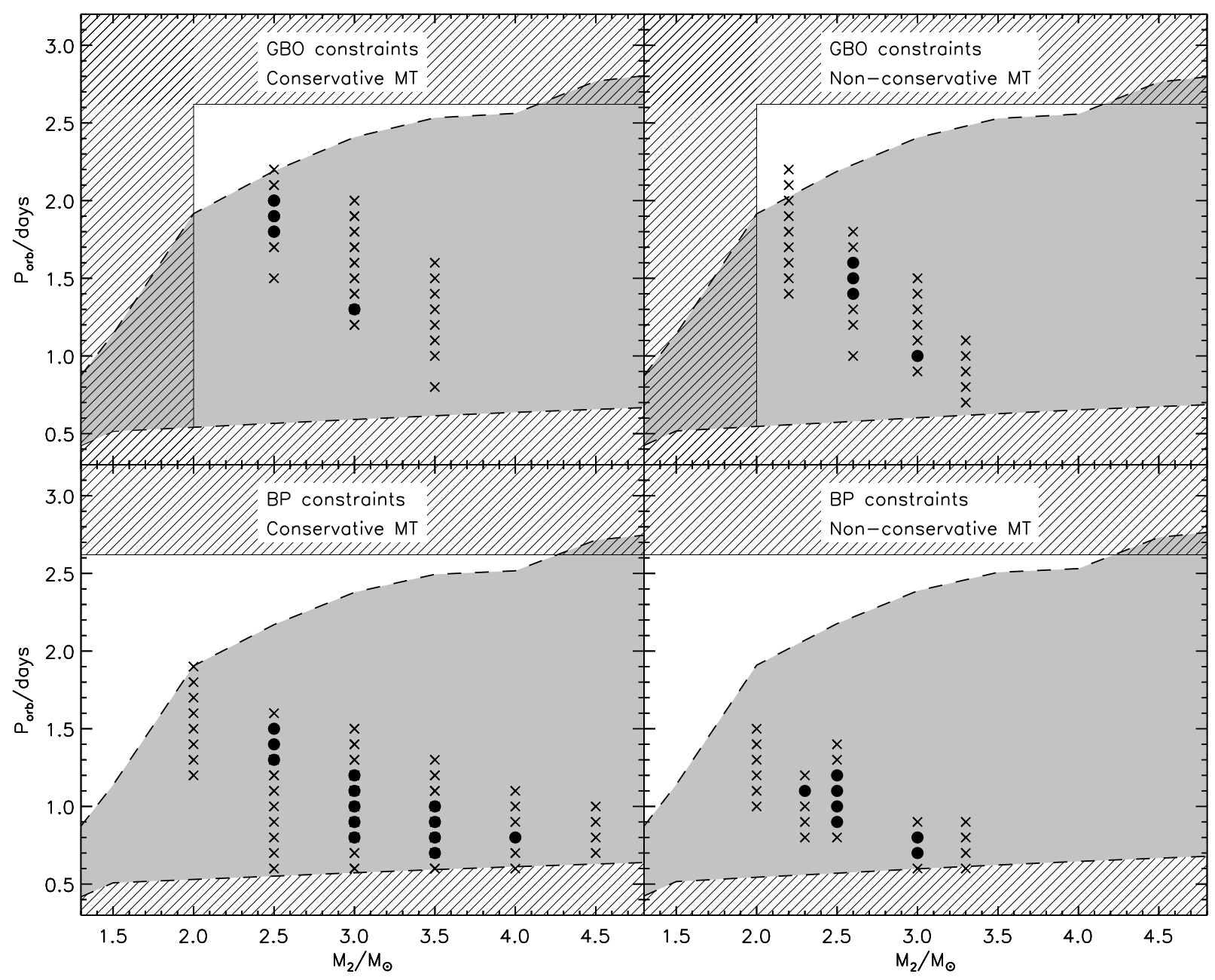

Fig. 2.- Initial donor masses and orbital periods of the evolutionary sequences at RLO onset. The grey shaded region delimited by the dashed lines indicates the region of the parameter space where the donor star is on the MS. Solid circles indicate MT sequences that are able to satisfy all the current observational constraints, while crosses indicate sequences that fail to do so. The hatched regions indicate regions of the parameter space that can be excluded without performing detailed MT calculations. For a given BH and donor mass, a lower limit on the initial period is given by the period at which the donor star fills its Roche lobe while it is still on the ZAMS. For MT sequences in which the orbital period increases as a result of MT, the current orbital period of 2.62 days furthermore provides an upper limit on the initial period. In the case of GBO constraints, a lower limit on the mass is given by the observational lower limit on the current donor mass of $2 M_{\odot}$. 
In the case of GBO constraints and conservative MT, successful sequences were found for initial $\mathrm{BH}$ masses ranging from $\simeq 5.5 M_{\odot}$ to $6.0 M_{\odot}$, initial donor masses ranging from $\simeq 2.5 M_{\odot}$ to $3.0 M_{\odot}$, and initial periods ranging from $\simeq 1.3$ to 2.0 days. The lower limit on the initial $\mathrm{BH}$ mass arises from the requirement that, for conservative MT, lower mass BHs need a more massive donor star in order to accrete enough matter to reach the observed present-day BH mass. For orbital periods where RLO occurs when the donor is still close to the ZAMS, the higher MT rates associated with more massive donor stars then cause the donor mass to decrease below the admissible range from 2.0 to2.8 $M_{\odot}$ before the period reaches the present-day value of 2.62 days. For longer orbital periods, on the other hand, the donor star has a somewhat more developed helium core at the onset of RLO, which causes the star to be too luminous when the system reaches the observed period of 2.62 days. The upper limit on the initial $\mathrm{BH}$ mass is determined by the requisite that as the initial $\mathrm{BH}$ mass gets closer to the present value of $6.3 \pm 0.5 M_{\odot}$, the donor star must start RLO closer to its present state as well. This eventually requires MT to start in the Hertzsprung gap which generally leads to MT rates that are too high for the system to be transient (see, however, Kolb et al. 1997 and Kolb 1998 for a discussion on soft X-ray transient in the Hertzsprung gap). In the case of non-conservative MT, we kept the BH mass fixed to the mean value of $6.3 M_{\odot}$. By keeping the $\mathrm{BH}$ mass constant we were able to to outline the initial donor masses at the onset of RLO with a somewhat higher resolution of 0.3 to $0.4 M_{\odot}$ without having to calculate an unrealistically large number of sequences. Successful sequences were found for $M_{2} \simeq 2.6-3.0 M_{\odot}$ and $P_{\text {orb }} \simeq 1.0-1.6$ days. When the donor star crosses the allowed region in the H-R diagram, all successful MT sequences found for the GBO system parameters are furthermore only marginally consistent with the currently observed orbital period and donor mass. The MT rates at the period of 2.62 days are always at least a factor of $\simeq 3$ smaller than the critical MT rate for transient behavior.

In the case of BP constraints and conservative MT, successful sequences were found for $M_{\mathrm{BH}} \simeq 3.5-4.5 M_{\odot}, M_{2} \simeq 2.5-4.0 M_{\odot}$, and $P_{\text {orb }} \simeq 0.7-1.5$ days, while for non-conservative MT successful sequences were found for $M_{\mathrm{BH}} \simeq 5.4 M_{\odot}, M_{2} \simeq 2.3 M_{\odot}-3.0 M_{\odot}$, and $P_{\text {orb }} \simeq$ $0.7-1.2$ days. The lower limit on the initial $\mathrm{BH}$ mass for conservative MT is again related to the higher donor masses required by initially less massive BHs to reach the present-day mass. The difference with the GBO sequences is, however, that the $\mathrm{BH}$ masses at the start of RLO are now so low that this requirement implies that MT initially starts on the thermal time scale of the donor star. This leads to highly super-Eddington MT rates and significant mass loss from the system. Consequently, initially even more massive donor stars are required for the $\mathrm{BH}$ to accrete enough to reach the observed present-day mass. These higher donor masses increase the MT rates even further, rendering the system persistent rather transient. The upper limit on the initial BH mass arises for the same reason as for the GBO sequences. 
The MT rates obtained when the donor star satisfies the observational constraints in the H-R diagram and when the binary reaches the observed period of 2.62 days span a wide range of values: for some sequences the MT rates are more than an order of magnitude smaller than the critical rate for transient behavior, while for others the system is only marginally transient. In general, however, it turned out to be easier to find MT sequences satisfying the BP constraints than sequences satisfying the GBO constraints.

An overview of the initial donor star masses and the initial orbital periods of the MT sequences listed in Tables $2-3$ is given in Fig. 2. Successful sequences are indicated by solid circles and unsuccessful ones by crosses. The figure clearly illustrates the extent of the parameter space covered by our calculations as well as the location of the successful sequences among the unsuccessful ones. The general trend in the initial parameters of the successful MT sequences is that lower initial donor masses require longer initial orbital periods in order to be successful. This is in agreement with the systematic behavior of the MT sequences illustrated in Fig. 1. We also note that MT sequences were calculated for BH masses outside the successful ranges quoted above, but for brevity these have been omitted from Tables 2-3 and Fig. 2.

Fig. 2 and Tables 2-3 also show there is no single unique MT sequence that satisfies all the observational constraints. Instead, a series of neighboring successful sequences is found for both GBO and BP constraints and for both conservative and non-conservative MT. In addition, the number of successful sequences found is limited only by our finite exploration of the available parameter space and the limitation of the calculations to the two extreme cases of fully conservative and fully non-conservative MT. Adopting a higher resolution grid of initial component masses and orbital periods would increase the number of successful sequences even further as would the consideration of MT sequences for which the degree of mass conservation is in between the two extreme cases of fully conservative or non-conservative MT. Fortunately, as we will see in Section 8, MT sequences with closely spaced initial parameters yield only small variations in the properties of the pre-SN binary progenitor and the kick that may have been imparted to the $\mathrm{BH}$ at birth. The vital point in the calculation of the presented MT sequences is therefore that we managed to outline the initial parameters of the successful sequences within a pre-determined accuracy rather than finding all possible MT sequences able to satisfy the present-day observational constraints for GRO J1655-40. 


\section{KINEMATIC HISTORY IN THE GALAXY}

Given the present position and velocity of GRO J1655-40 in the Galaxy, we derive the post-SN peculiar velocity of the binary's mass center by tracing its orbit in the Galaxy back to the birth time of the $\mathrm{BH}$. A specific birth time is derived for each successful MT sequence by setting it equal to the current age of the donor star. The latter is a good estimate of the $\mathrm{BH}$ birth time within an uncertainty equal to the lifetime of the massive progenitor of the $\mathrm{BH}$, which is typically less than $10 \mathrm{Myr}$. This uncertainty is always much smaller than the actual time expired since the birth of the $\mathrm{BH}$.

We describe the motion of the system with respect to a right-handed Cartesian frame of reference $O X Y Z$ whose origin coincides with the Galactic center and whose $X Y$-plane coincides with the mid-plane of the Galactic disk. The direction from the projection of the Sun's position onto the Galactic plane to the Galactic center is taken as the positive direction of the $X$-axis, the direction from the Sun to the Northern Galactic pole as the positive direction of the $Z$-axis, and the direction of the Galactic rotational velocity at the position of the Sun as the positive direction of the $Y$-axis. In terms of these coordinates, the Sun is located at $\left(X_{\odot}, Y_{\odot}, Z_{\odot}\right)$ with $X_{\odot}=-8 \pm 0.5 \mathrm{kpc}, Y_{\odot}=0$, and $Z_{\odot}=30 \pm 25 \mathrm{pc}$ (Reid 1993; Humphreys \& Larsen 1995, and references therein). GRO J1655-40 is currently located at a distance from the Sun of $3.2 \pm 0.2 \mathrm{kpc}$ (Hjellming \& Rupen 1995), a Galactic longitude $l=345.0^{\circ}$, and a Galactic latitude $b=+2.2^{\circ}$ (Tingay et al. 1995); corresponding to $X=-4.9 \pm 0.5 \mathrm{kpc}, Y=-0.83 \pm 0.05 \mathrm{kpc}$, and $Z=0.15 \pm 0.03 \mathrm{kpc}$. The velocity components of GRO J1655-40 with respect to the $X$-, $Y$-, and $Z$-axes are $U=-121 \pm 18 \mathrm{~km} \mathrm{~s}^{-1}, V=$ $-33 \pm 8 \mathrm{~km} \mathrm{~s}^{-1}$, and $W=3 \pm 8 \mathrm{~km} \mathrm{~s}^{-1}$ (Mirabel et al. 2002).

To model the Galaxy, we adopt the Galactic potential of Carlberg \& Innanen (1987) with updated model parameters of Kuijken \& Gilmore (1989). For each successful MT sequence (marked in boldface in Tables 2 and 3), the equations governing the system's motion in the Galaxy are integrated backward in time up to the time corresponding to the current age of the donor star, as given by the evolutionary sequence. 

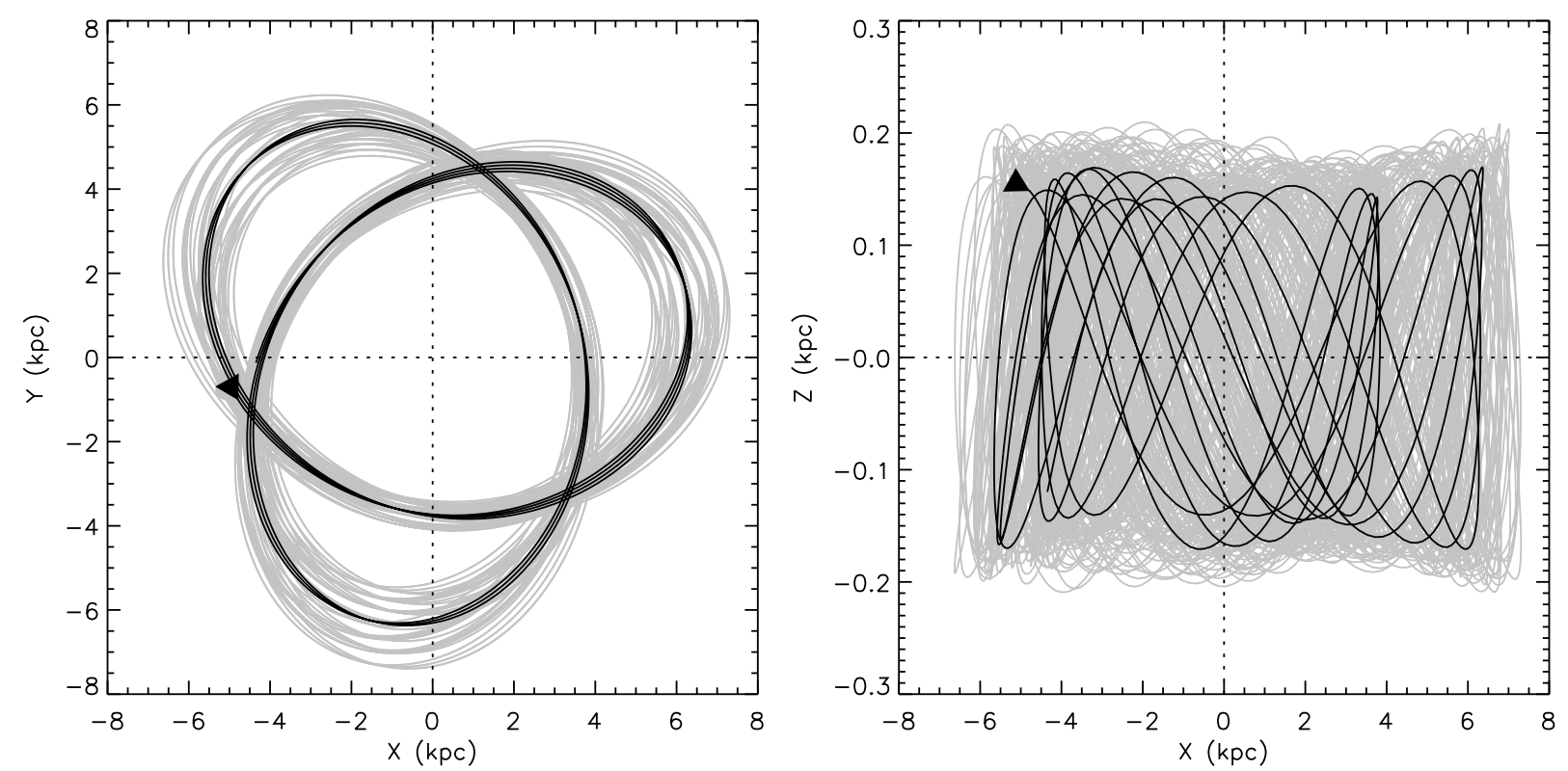

Fig. 3.- Orbit of GRO J1655-40 in the Galaxy up to $1 \mathrm{Gr}$ in the past. In the left-hand panel, the orbit is shown projected on the $X Y$-plane, while in the right-hand panel it is projected on the $X Z$-plane (see text for a definition of the coordinate axes). The black line indicates the orbit for a present-day distance of $3.2 \mathrm{kpc}$ and velocity components $U=-121 \mathrm{~km} \mathrm{~s}^{-1}$, $V=-33 \mathrm{~km} \mathrm{~s}^{-1}$, and $W=3 \mathrm{~km} \mathrm{~s}^{-1}$. The grey lines indicate the uncertainties in the orbit associated with the error bars in the distance and the velocity components. The arrow indicates the present-day position of GRO J1655-40 and the direction of the motion backward in time. 
In Fig. 3, we show the orbit of GRO J1655-40 projected on the $X Y$ - and $X Z$-planes up to $1 \mathrm{Gyr}$ in the past, which encompasses the entire range of donor ages from $\simeq 335$ to $\simeq 998$ Myr given by the successful MT sequences. The black line represents the orbit obtained for the mean values of the distance and velocity components, while the grey lines indicate the deviations from this orbit obtained from considering all possible combinations of the extreme values of the distance and velocity component measurements (i.e. the endpoints of the error bars). In agreement with the findings of Mirabel et al. (2002), the system always remains within 3 to $7 \mathrm{kpc}$ from the Galactic center and within 200 pc from the Galactic plane. 


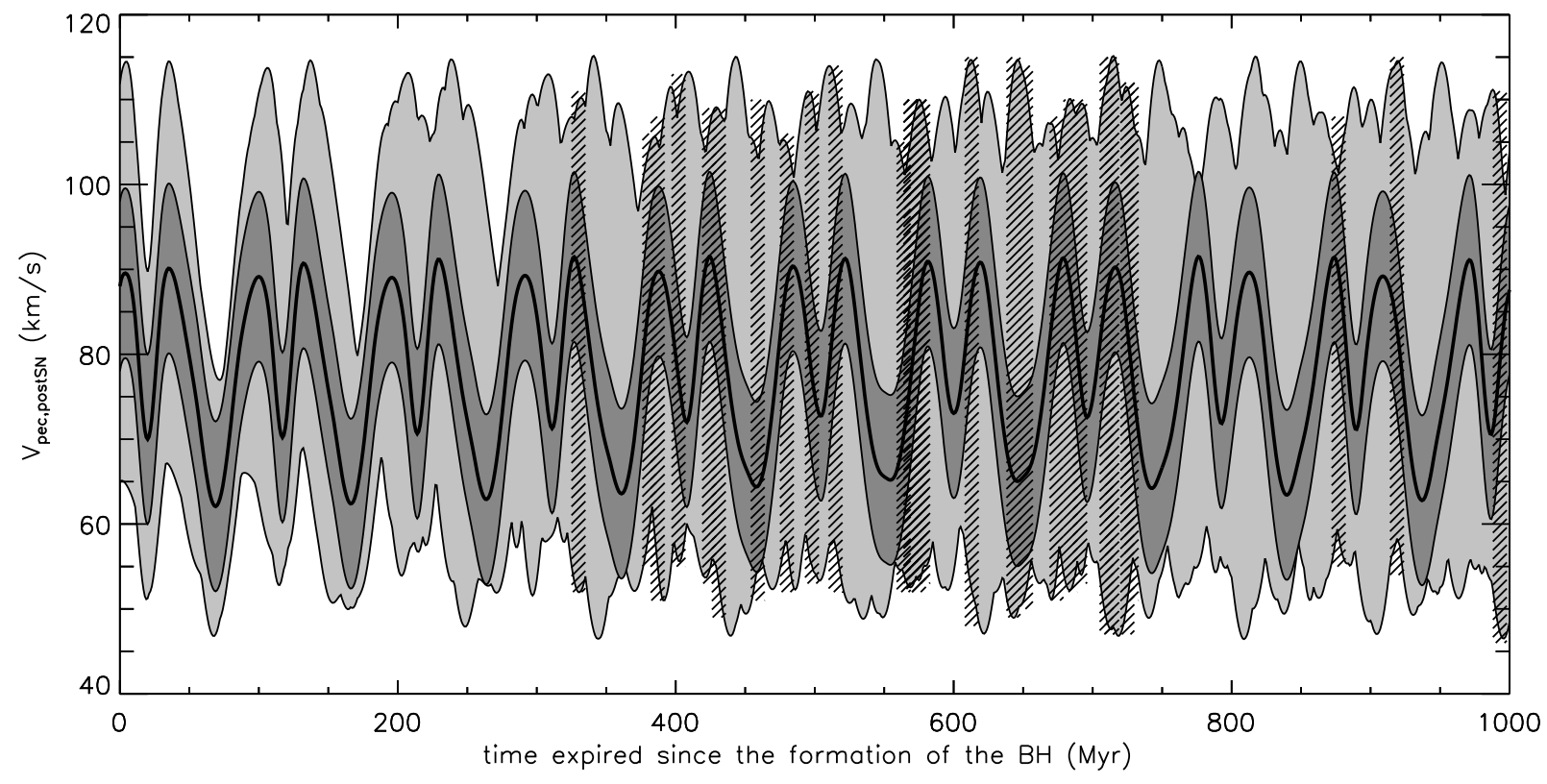

Fig. 4.- Post-SN peculiar velocity of GRO J1655-40 as a function of the time expired since the formation of the BH. The black solid line represents the post-SN peculiar velocity for the mean values of the binary's current distance and 3D velocity, and the light grey area indicates the uncertainties resulting from the error bars in the distance and the velocity components. The dark grey area indicates the $\pm 10 \mathrm{~km} \mathrm{~s}^{-1}$ errors bars on $V_{\text {pec,postSN }}$. Possible birth times of the BH given by the successful MT sequences listed in Tables 2 and 3 are indicated by the hatched vertical bars. 
The post-SN peculiar velocity $V_{\text {pec,postSN }}$ of the binary is obtained by subtracting the local Galactic rotational velocity from the total systemic velocity at the birth site of the $\mathrm{BH}$. The variations of $V_{\text {pec,postSN }}$ as a function of the time expired since the formation of the $\mathrm{BH}$ are displayed in Fig. 4. The hatched vertical bars indicate the BH birth times determined by the age of the donor star for each of the successful MT sequences listed in Tables 2-3. The width of the bars corresponds to an uncertainty in the birth times of $10 \mathrm{Myr}$. When accounting for the uncertainties in the distance and the velocity components (cf. Fig. 3), the post-SN peculiar velocity can span a wide range of values ranging from $\simeq 45$ to $\simeq 115 \mathrm{~km} \mathrm{~s}^{-1}$. This wide range is a result of our determination of the possible uncertainties in the Galactic

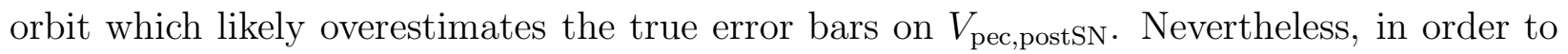
be conservative, we consider the full range of possible post-SN peculiar velocities given by the grey shaded area in Fig. 4.

\section{ORBITAL EVOLUTION DUE TO TIDES AND GENERAL RELATIVITY}

After the formation of the $\mathrm{BH}$, the orbital parameters of the binary are subjected to secular changes due to the perturbation of the external gravitational field from spherical symmetry by the MS star's tidal distortion and due to the loss of orbital angular momentum via gravitational radiation. Since the tidal interactions depend on both the orbital and rotational properties of the MS star, the star's rotational angular velocity right after the SN explosion that formed the BH enters the problem as an additional unknown quantity. We here assume that the the rotational angular velocity of the MS star is unaffected by the SN explosion and that just before the explosion the star's rotation rate was synchronized with the orbital motion. The post-SN rotational angular velocity is then given by $\Omega_{\text {postSN }}=$ $2 \pi / P_{\text {orb,presn }}$, where $P_{\text {orb,preSN }}$ is the pre-SN orbital period. As a test, we also considered the more extreme cases $\Omega_{\text {postSN }}=0$ and $\Omega_{\text {postSN }}=10 \times 2 \pi / P_{\text {orb,preSN }}$, but these were found to not affect significantly the outcome of the orbital evolution between the formation of the $\mathrm{BH}$ and the start of the RLO phase.

We adopt the system of equations governing the tidal evolution of the semi-major axis $A$, the orbital eccentricity $e$, and the MS star's rotational angular velocity $\Omega$ from Hut (1981):

$$
\begin{aligned}
& \left(\frac{d A}{d t}\right)_{\mathrm{tides}}=-6 \frac{k_{2}}{T} \frac{M_{\mathrm{BH}}}{M_{2}} \frac{M_{\mathrm{BH}}+M_{2}}{M_{2}}\left(\frac{R_{2}}{A}\right)^{8} \\
& \times \frac{A}{\left(1-e^{2}\right)^{15 / 2}}\left[f_{1}\left(e^{2}\right)-\left(1-e^{2}\right)^{3 / 2} f_{2}\left(e^{2}\right) \frac{\Omega}{n}\right],
\end{aligned}
$$




$$
\begin{gathered}
\left(\frac{d e}{d t}\right)_{\text {tides }}=-27 \frac{k_{2}}{T} \frac{M_{\mathrm{BH}}}{M_{2}} \frac{M_{\mathrm{BH}}+M_{2}}{M_{2}}\left(\frac{R_{2}}{A}\right)^{8} \\
\times \frac{e}{\left(1-e^{2}\right)^{13 / 2}}\left[f_{3}\left(e^{2}\right)-\frac{11}{18}\left(1-e^{2}\right)^{3 / 2} f_{4}\left(e^{2}\right) \frac{\Omega}{n}\right], \\
\left(\frac{d \Omega}{d t}\right)_{\text {tides }}=3 \frac{k_{2}}{T}\left(\frac{M_{\mathrm{BH}}}{M_{2}}\right)^{2} \frac{M_{2} R_{2}^{2}}{I_{2}}\left(\frac{R_{2}}{A}\right)^{6} \\
\times \frac{n}{\left(1-e^{2}\right)^{6}}\left[f_{2}\left(e^{2}\right)-\left(1-e^{2}\right)^{3 / 2} f_{5}\left(e^{2}\right) \frac{\Omega}{n}\right]
\end{gathered}
$$

where

$$
\begin{gathered}
f_{1}\left(e^{2}\right)=1+\frac{31}{2} e^{2}+\frac{255}{8} e^{4}+\frac{185}{16} e^{6}+\frac{25}{64} e^{8}, \\
f_{2}\left(e^{2}\right)=1+\frac{15}{2} e^{2}+\frac{45}{8} e^{4}+\frac{5}{16} e^{6} \\
f_{3}\left(e^{2}\right)=1+\frac{15}{4} e^{2}+\frac{15}{8} e^{4}+\frac{5}{64} e^{6} \\
f_{4}\left(e^{2}\right)=1+\frac{3}{2} e^{2}+\frac{1}{8} e^{4} \\
f_{5}\left(e^{2}\right)=1+3 e^{2}+\frac{3}{8} e^{4}
\end{gathered}
$$

(see also Zahn 1977, 1978). In these equations, $M_{\mathrm{BH}}$ is the mass of the $\mathrm{BH} ; M_{2}, R_{2}, k_{2}$, and $I_{2}$ are the mass, radius, apsidal-motion constant, and moment of inertia of the companion star, $n=2 \pi / P_{\text {orb }}$ is the mean orbital angular velocity, and $T$ is a characteristic time scale for the orbital evolution due to tides.

For stars with radiative envelopes, as we are dealing with here, the dominant mechanism contributing to the dissipative tidal forces is radiative damping of dynamical tides (Zahn $1975,1977)$. The factor $k_{2} / T$ can then be approximated as

$$
\begin{aligned}
\left(\frac{k_{2}}{T}\right)_{\mathrm{rad}} & =1.9782 \times 10^{4} f_{\mathrm{cal}}\left(\frac{R_{2}}{R_{\odot}}\right)^{2}\left(\frac{R_{\odot}}{A}\right) \\
& \times\left(\frac{M_{2}}{M_{\odot}}\right)\left(\frac{M_{\mathrm{BH}}+M_{2}}{M_{2}}\right)^{5 / 6} E_{2} \mathrm{yr}^{-1}
\end{aligned}
$$

with

$$
E_{2}=1.592 \times 10^{-9}\left(\frac{M_{2}}{M_{\odot}}\right)^{2.84}
$$

(Hurley, Tout, \& Pols 2002). 
Eqs. (10) and (11) are strictly speaking only valid for dynamic tides with low forcing frequencies that are far away from any of the eigenfrequencies of the star's free modes of oscillation. Witte \& Savonije (1999) have shown that in close binaries with eccentric orbits the system can easily become locked in a resonance between a dynamic tide and a free oscillation mode and that such resonance lockings can greatly accelerate the orbital evolution. In the expression for $k_{2} / T$, we therefore incorporated the calibration factor $f_{\text {cal }}$ to account for some of the still existing uncertainties in the strength of tidal dissipation in stars containing radiative envelopes. In our calculations, we adopt $f_{\text {cal }}=10$ which has been shown to be appropriate for consistency with both the observations of circularization periods in open clusters and with orbital decay rates measured in high-mass X-ray binaries (for more details see Belczynski et al. 2004).

To follow the secular changes in the orbital parameters associated with the emission of gravitational waves, we adopt the system of equations given by Junker \& Schäfer (1992):

$$
\begin{aligned}
\left(\frac{d A}{d t}\right)_{\mathrm{GR}} & =-\frac{2 c}{15} \frac{\nu}{\left(1-e^{2}\right)^{9 / 2}}\left[\frac{G\left(M_{\mathrm{BH}}+M_{2}\right)}{A c^{2}}\right]^{3} \\
& \times\left(96+292 e^{2}+37 e^{4}\right)\left(1-e^{2}\right) \\
\left(\frac{d e}{d t}\right)_{\mathrm{GR}} & =-\frac{1}{15} \frac{\nu c^{3}}{G\left(M_{\mathrm{BH}}+M_{2}\right)}\left[\frac{G\left(M_{\mathrm{BH}}+M_{2}\right)}{A c^{2}}\right]^{4} \\
& \times \frac{e\left(304+121 e^{2}\right)\left(1-e^{2}\right)}{\left(1-e^{2}\right)^{7 / 2}} .
\end{aligned}
$$

Here, $G$ is the Newtonian gravitational constant, $c$ is the speed of light, and $\nu=M_{\mathrm{BH}} M_{2} /\left(M_{\mathrm{BH}}+\right.$ $\left.M_{2}\right)^{2}$. For brevity, we restricted Eqs. (12) and (13) to the lowest-order terms in the small parameter $G\left(M_{\mathrm{BH}}+M_{2}\right) /\left(A c^{2}\right)$. In the numerical calculations however, we adopt the full 3.5 post-Newtonian order equations derived by Junker \& Schäfer (1992).

The orbital evolution due to tides and gravitational radiation is illustrated in Fig. 5 for the example case of a binary consisting of a $4.5 M_{\odot} \mathrm{BH}$ and a $2.5 M_{\odot}$ zero-age MS star, and for four different combinations of initial orbital separations and eccentricities. The four combinations are chosen such that after approximately $\simeq 480 \mathrm{Myr}$ of orbital evolution the total angular momentum of the binary is approximately equal to the total angular momentum of the binary represented by MT sequence BP62 (see Table 3) at the onset of the MT phase ${ }^{3}$.

\footnotetext{
${ }^{3}$ Note that $480 \mathrm{Myr}$ corresponds to the age of the donor star at the onset of MT in sequence BP62. The choice to match the orbital angular momentum at the end of the orbital evolution calculations to the orbital angular momentum of the MT sequence at the onset of RLO is equivalent to assuming that tides circularize the binary instantaneously at $t \simeq 480 \mathrm{Myr}$.
} 

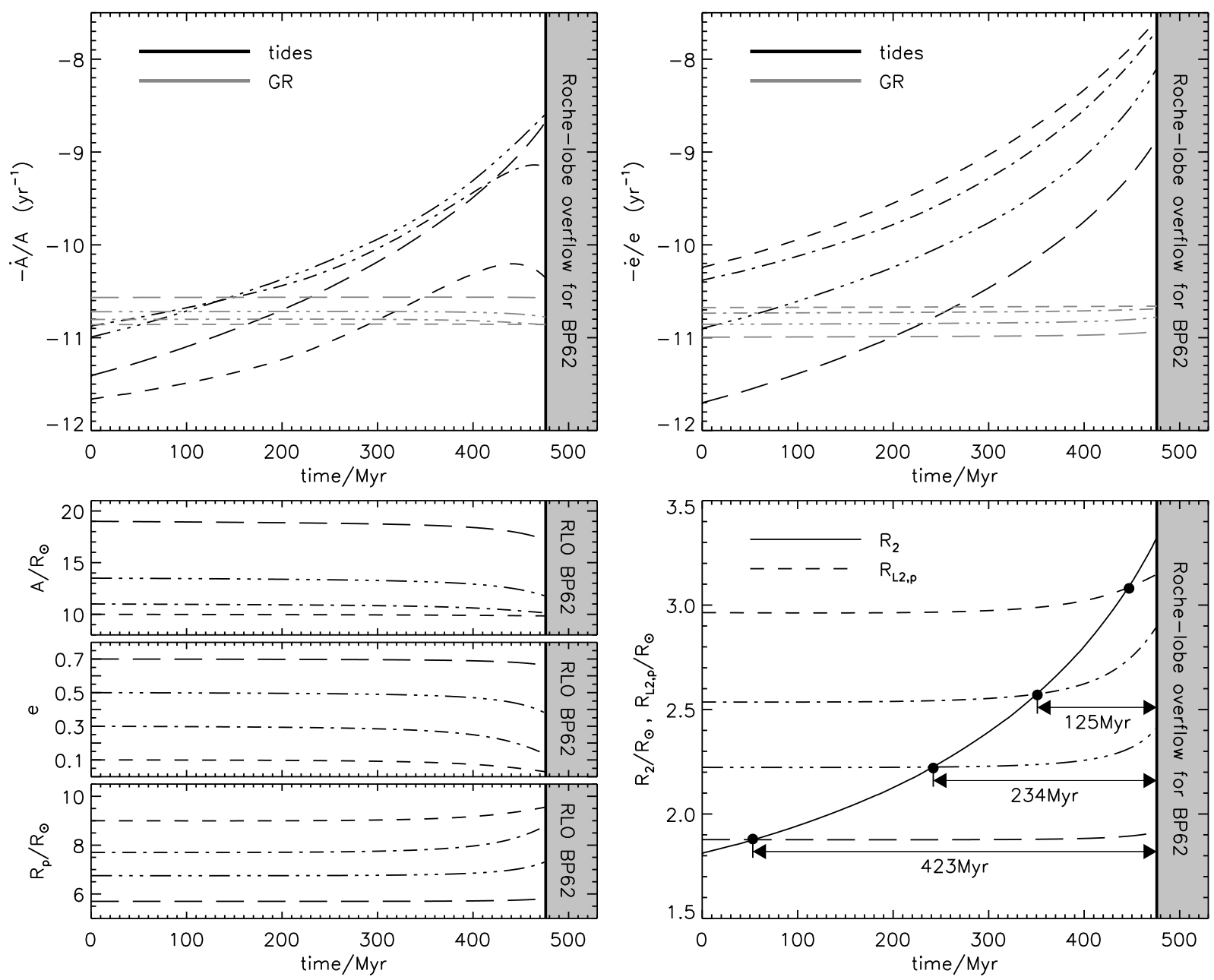

Fig. 5.- Secular evolution of a binary consisting of a $4.5 M_{\odot} \mathrm{BH}$ and a $2.5 M_{\odot}$ ZAMS star, for four different combinations of initial orbital separations and eccentricities (see the bottom left panels to identify the combinations and the associated line types). The top panels show the relative rates of secular change of the semi-major axis $A$ and the orbital eccentricity $e$ as a result of the MS star's tidal distortion and gravitational radiation separately. The bottom-left panels show the evolution of the semi-major axis, orbital eccentricity, and periastron distance $R_{p}=A(1-e)$ resulting from the combined effects of tides and gravitational radiation, while the bottom-right panel shows the associated evolution of the MS star's Roche-lobe radius at the periastron of its relative orbit. For comparison, the evolution of the star's radius is also shown in the bottom-right panel. The thick vertical lines followed by the grey-shaded areas mark the beginning of the RLO phase for the successful MT sequence BP62. 
The two upper panels of Fig. 5 show the relative rates of secular change of the semi-major axis $A$ and the orbital eccentricity $e$ (for tides and GR separately) as functions of the time expired since the formation of the BH. For all four combinations of initial orbital separations and eccentricities, the GR rates remain almost constant throughout the considered time interval, while the rates due to tides increase significantly as the star evolves on the MS. This behavior reflects the independence of gravitational radiation on the size and structure of the star and the strong dependence of tides on the ratio of the stellar radius to the orbital semi-major axis. The plots also illustrate that, for binaries consisting of a compact object and a young early-type secondary, gravitational radiation and tides, at some points in time, may affect the orbital evolution to comparable degrees.

The actual evolution of the semi-major axis $A$, the orbital eccentricity $e$, and the periastron distance $R_{p}=A(1-e)$ due to the combined effect of tides and gravitational radiation is displayed in the lower-left panels of Fig. 5. As expected from the low rates of secular change displayed in the upper two panels, the orbital parameters remain almost constant during the first 300-400 Myr of evolution and start changing noticeably when the star gets closer to the end of the MS and tidal effects become dominant.

Finally, in the lower-right panel of Fig. 5 we compare the evolution of the stellar radius with the evolution of the Roche-lobe radius at the periastron of the MS star's relative orbit for each of the four considered combinations of initial orbital separations and eccentricities. The Roche-lobe radii at periastron are determined by

$$
\frac{R_{\mathrm{Lp}, 2}}{R_{p}}=\frac{0.49 q^{2 / 3}}{0.6 q^{2 / 3}+\ln \left(1+q^{1 / 3}\right)},
$$

where $q=M_{2} / M_{\mathrm{BH}}$ (Eggleton 1983). It follows that although all four pairs of initial orbital separations and eccentricities lead to orbital configurations that are consistent with the successful MT sequence BP62 at the onset of RLO, only one pair ( $\left.A=10 R_{\odot}, e=0.1\right)$ leads to RLO at periastron at a time that is sufficiently close to the age of the donor star required to make the sequence successful in the first place. The other three pairs all yield RLO at periastron at times that are $\simeq 125-420 \mathrm{Myr}$ too early. They would therefore only yield viable progenitors for GRO J1655-40 if RLO at periastron is assumed to conserve the binary's total angular momentum and if the total amount of mass transferred from the donor star is small enough to not significantly change its properties so that it would follow a different evolutionary path in the H-R diagram. If either of these conditions is violated, the considered pair of initial orbital separation and eccentricity is incompatible with the starting point of the successful RLO sequence under consideration. 


\section{ORBITAL DYNAMICS AT CORE COLLAPSE}

At the time of the SN explosion, the mass lost from the system and possibly the kick imparted to the BH during the explosion change the binary's orbital parameters. The preand post-SN component masses, orbital semi-major axis, and orbital eccentricity are related by the conservation laws of orbital energy and angular momentum, which in turn depend on the magnitude and the direction of the kick velocity that may be imparted to the $\mathrm{BH}$. We describe these relations and the associated progenitor and kick velocity constraints in more detail in Sections 7.1 and 7.2. In these sections, we adopt the same notations for the orbital elements as before, but add the subscripts "preSN" and "postSN" to distinguish between their values before and after the SN explosion that formed the BH.

We first note though that there are two additional constraints that need to be imposed on the $\mathrm{BH}$ progenitor besides those arising from the orbital dynamics of symmetric and asymmetric SN explosions. One is that, for each successful MT sequence, the progenitor mass must of course be more massive than the initial BH mass at the onset of RLO. The other is that the BH progenitor must fit within its pre-SN Roche lobe. Strictly speaking this is not a necessary condition (Dewi \& van den Heuvel 2004; Willems \& Kalogera 2004; and Willems et al. 2004, for example, showed that the progenitor of PSR J0737-3039B was filling its Roche lobe at the time of its collapse). Nevertheless, we impose this condition to avoid complications arising from accretion-induced changes in the stellar structure of the MS companion that will become the XRB donor star during the X-ray phase. We have tested this assumption by omitting the second constraint from our calculations and found no appreciable change in the derived progenitor and kick velocity constraints.

\subsection{Symmetric SN explosions}

The orbital dynamics of symmetric SN explosions have been discussed extensively in the past (e.g., Blaauw 1961; Boersma 1961; Hills 1983; Fryer \& Kalogera 1997). We recall that the changes in the pre-SN orbital parameters are entirely determined by the amount of mass lost from the system during the explosion, yielding a post-SN semi-major axis and orbital eccentricity given by

$$
\begin{gathered}
\frac{A_{\text {postSN }}}{A_{\text {preSN }}}=\frac{M_{\mathrm{BH}}+M_{2}}{M_{2}+2 M_{\mathrm{BH}}-M_{\mathrm{He}}}, \\
e_{\mathrm{postSN}}=\frac{M_{\mathrm{He}}-M_{\mathrm{BH}}}{M_{\mathrm{BH}}+M_{2}},
\end{gathered}
$$

where $M_{\mathrm{He}}$ is the mass of the BH's helium star progenitor. 
The mass loss from the system also imparts a kick to the binary's center of mass with a magnitude equal to

$$
V_{\mathrm{pec}, \text { postSN }}=\frac{\left(M_{\mathrm{He}}-M_{\mathrm{BH}}\right) M_{2}}{\left(M_{\mathrm{BH}}+M_{2}\right)\left(M_{\mathrm{He}}+M_{2}\right)} V_{\mathrm{He}, \mathrm{preSN}}
$$

where

$$
V_{\mathrm{He}, \mathrm{preSN}}=\left[\frac{G\left(M_{\mathrm{He}}+M_{2}\right)}{A_{\mathrm{preSN}}}\right]^{1 / 2}
$$

is the relative orbital velocity of the helium star just before its instantaneous SN explosion.

\subsection{Asymmetric SN explosions}

For asymmetric SN explosions, the newly formed BH receives a kick at birth, the magnitude and direction of which can either counteract or reinforce the effect of the mass lost from the system. The pre- and post-SN binary parameters are related by the equations

$$
\begin{gathered}
V_{\mathrm{k}}^{2}+V_{\mathrm{He}, \mathrm{preSN}}^{2}+2 V_{\mathrm{k}} V_{\mathrm{He}, \mathrm{preSN}} \cos \theta \\
=G\left(M_{\mathrm{BH}}+M_{2}\right)\left(\frac{2}{A_{\mathrm{preSN}}}-\frac{1}{A_{\mathrm{postSN}}}\right), \\
A_{\mathrm{preSN}}^{2}\left[V_{\mathrm{k}}^{2} \sin ^{2} \theta \cos ^{2} \phi+\left(V_{\mathrm{k}} \cos \theta+V_{\mathrm{He}, \mathrm{preSN}}\right)^{2}\right] \\
=G\left(M_{\mathrm{BH}}+M_{2}\right) A_{\mathrm{postSN}}\left(1-e_{\mathrm{postSN}}^{2}\right)
\end{gathered}
$$

(e.g., Hills 1983; Brandt \& Podsiadlowski 1995; Kalogera 1996; Fryer \& Kalogera 1997; Kalogera \& Lorimer 2000). Here, $V_{k}$ is the magnitude of the kick velocity, $\theta$ is the polar angle between the kick velocity and the relative orbital velocity of the helium star just before the SN explosion, and $\phi$ is the corresponding azimuthal angle defined so that $\phi=0$ represents a plane perpendicular to the line connecting the centers of mass of the binary components (see Fig. 1 in Kalogera 2000 for a graphical representation).

The requirements that the binary must remain bound after the SN explosion and that the direction of the kick must be real $\left(0 \leq \sin ^{2} \theta \leq 1\right.$ and $\left.0 \leq \cos ^{2} \phi \leq 1\right)$ impose constraints on the pre- and post-SN parameters and on the magnitude of the kick velocity imparted to the $\mathrm{BH}$ at birth. In particular, the ratio of the pre- to post-SN semi-major axes must satisfy the inequalities

$$
1-e_{\mathrm{postSN}} \leq \frac{A_{\mathrm{preSN}}}{A_{\mathrm{postSN}}} \leq 1-e_{\mathrm{postSN}}
$$




$$
\begin{aligned}
& \frac{A_{\mathrm{preSN}}}{A_{\mathrm{postSN}}}<2-\frac{M_{\mathrm{He}}+M_{2}}{M_{\mathrm{BH}}+M_{2}}\left(\frac{V_{k}}{V_{\mathrm{He}, \mathrm{preSN}}}-1\right)^{2}, \\
& \frac{A_{\mathrm{preSN}}}{A_{\mathrm{postSN}}}>2-\frac{M_{\mathrm{He}}+M_{2}}{M_{\mathrm{BH}}+M_{2}}\left(\frac{V_{k}}{V_{\mathrm{He}, \mathrm{preSN}}}+1\right)^{2} .
\end{aligned}
$$

The first of these inequalities expresses the condition that the post-SN orbit must pass through the position of the two stars at the time of the SN explosion (Flannery \& van den Heuvel 1975). The last two inequalities correspond to lower and upper limits on the amount of orbital contraction or expansion that can take place for a given amount of mass loss and a given magnitude of the kick velocity (see, e.g., Kalogera \& Lorimer 2000).

The magnitude of the kick velocity imparted to the $\mathrm{BH}$ at birth is restricted to the range determined by (Brandt \& Podsiadlowski 1995; Kalogera \& Lorimer 2000)

$$
\begin{aligned}
& \frac{V_{k}}{V_{\mathrm{He}, \mathrm{preSN}}}<1+\left(2 \frac{M_{\mathrm{BH}}+M_{2}}{M_{\mathrm{He}}+M_{2}}\right)^{1 / 2}, \\
& \frac{V_{k}}{V_{\mathrm{He}, \text { preSN }}}>1-\left(2 \frac{M_{\mathrm{BH}}+M_{2}}{M_{\mathrm{He}}+M_{2}}\right)^{1 / 2} .
\end{aligned}
$$

The first inequality expresses the requirement that the binary must remain bound after the SN explosion, while the second inequality yields the minimum kick velocity required to keep the system bound if more than half of the total system mass is lost in the explosion.

Last, an upper limit on the mass of the BH progenitor can be derived from the condition that the azimuthal direction of the kick is real, i.e., $\cos ^{2} \phi \geq 0$ (Fryer \& Kalogera 1997):

$$
\begin{aligned}
M_{\mathrm{He}} & \leq-M_{2}+k^{2}\left(M_{2}+M_{\mathrm{BH}}\right) \frac{A_{\mathrm{preSN}}}{A_{\mathrm{postSN}}} \\
& \times\left\{2\left(\frac{A_{\mathrm{postSN}}}{A_{\mathrm{preSN}}}\right)^{2}\left(1-e_{\mathrm{postSN}}^{2}\right)-k\right. \\
& -2 \frac{A_{\mathrm{postSN}}}{A_{\mathrm{preSN}}}\left(1-e^{2}\right)^{1 / 2} \\
& \left.\times\left[\left(\frac{A_{\mathrm{postSN}}}{A_{\mathrm{preSN}}}\right)^{2}\left(1-e_{\mathrm{postSN}}^{2}\right)-k\right]^{1 / 2}\right\}^{-1},
\end{aligned}
$$

where

$$
k=2 \frac{A_{\text {postSN }}}{A_{\text {preSN }}}-\left[\frac{V_{\mathrm{k}}^{2} A_{\text {postSN }}}{G\left(M_{2}+M_{\mathrm{BH}}\right)}+1\right] .
$$

From Eqs. (3) and (34) in Kalogera (1996), it also follows that the mass loss and kick experienced during the SN explosion yield a post-SN peculiar velocity for the binary's center 
of mass determined by

$$
V_{\text {pec,postSN }}^{2}=\nu_{1} V_{\mathrm{He}, \mathrm{preSN}}^{2}+\nu_{2} V_{\mathrm{BH}, \mathrm{postSN}}^{2}+\nu_{3} V_{k}^{2},
$$

where

$$
V_{\mathrm{BH}, \mathrm{postSN}}^{2}=G\left(M_{\mathrm{BH}}+M_{2}\right)\left(\frac{2}{A_{\text {preSN }}}-\frac{1}{A_{\text {postSN }}}\right)
$$

is the square of the relative orbital velocity of the $\mathrm{BH}$ immediately after the SN explosion. The coefficients $\nu_{1}, \nu_{2}$, and $\nu_{3}$ are defined as

$$
\begin{gathered}
\nu_{1}=\frac{M_{\mathrm{He}} M_{2}\left(M_{\mathrm{He}}-M_{\mathrm{BH}}\right)}{\left(M_{\mathrm{BH}}+M_{2}\right)\left(M_{\mathrm{He}}+M_{2}\right)^{2}}, \\
\nu_{2}=-\frac{M_{\mathrm{BH}} M_{2}\left(M_{\mathrm{He}}-M_{\mathrm{BH}}\right)}{\left(M_{\mathrm{BH}}+M_{2}\right)^{2}\left(M_{\mathrm{He}}+M_{2}\right)}, \\
\nu_{3}=\frac{M_{\mathrm{BH}} M_{\mathrm{He}}}{\left(M_{\mathrm{BH}}+M_{2}\right)\left(M_{\mathrm{He}}+M_{2}\right)} .
\end{gathered}
$$

For each successful MT sequence, we use the post-SN orbital parameters derived by following the Galactic motion and the orbital evolution due to tides and gravitation radiation backward in time (see Sections 5 and 6) and explore the parameter space made up by $A_{\text {preSN }}$, $M_{\mathrm{He}}, V_{k}, \theta$, and $\phi$ to look for pre-SN binary configurations and kick parameters satisfying Eqs. (19)-(32), or, in the case of $V_{k}=0$, Eqs. (15)-(18). As we will see in the next section, this allows us to identify well defined regions of progenitor and kick properties that are fully consistent with the complete set of observational constraints described in Section 2.

\section{PROGENITOR CONSTRAINTS}

The elements presented in the previous sections can now be combined to establish a complete picture of the evolutionary history of GRO J1655-40, the pre- and post-SN binary properties, and the dynamics involved in the core-collapse event that formed the BH. For each of the MT sequences that reproduce all of the system's currently observed properties, we first trace both the motion of the system in the Galaxy and the orbital evolution due to tides and gravitational radiation back in time to the formation of the $\mathrm{BH}$. The $\mathrm{BH}$ formation time is different for each MT sequence and is estimated by the age of the donor star. This procedure gives us the binary's peculiar velocity and orbital parameters right after the $\mathrm{BH}$ formation. We then use the conservation laws of orbital energy and orbital angular momentum together with all the associated orbital dynamics constraints at core collapse to link the pre-SN binary properties and the possible BH kick to the post-SN binary properties. In the following 
paragraphs, we first, as an example, discuss the progenitor constraints derived following this procedure for a single successful MT sequence, and next summarize the conclusions from all successful MT sequences. 


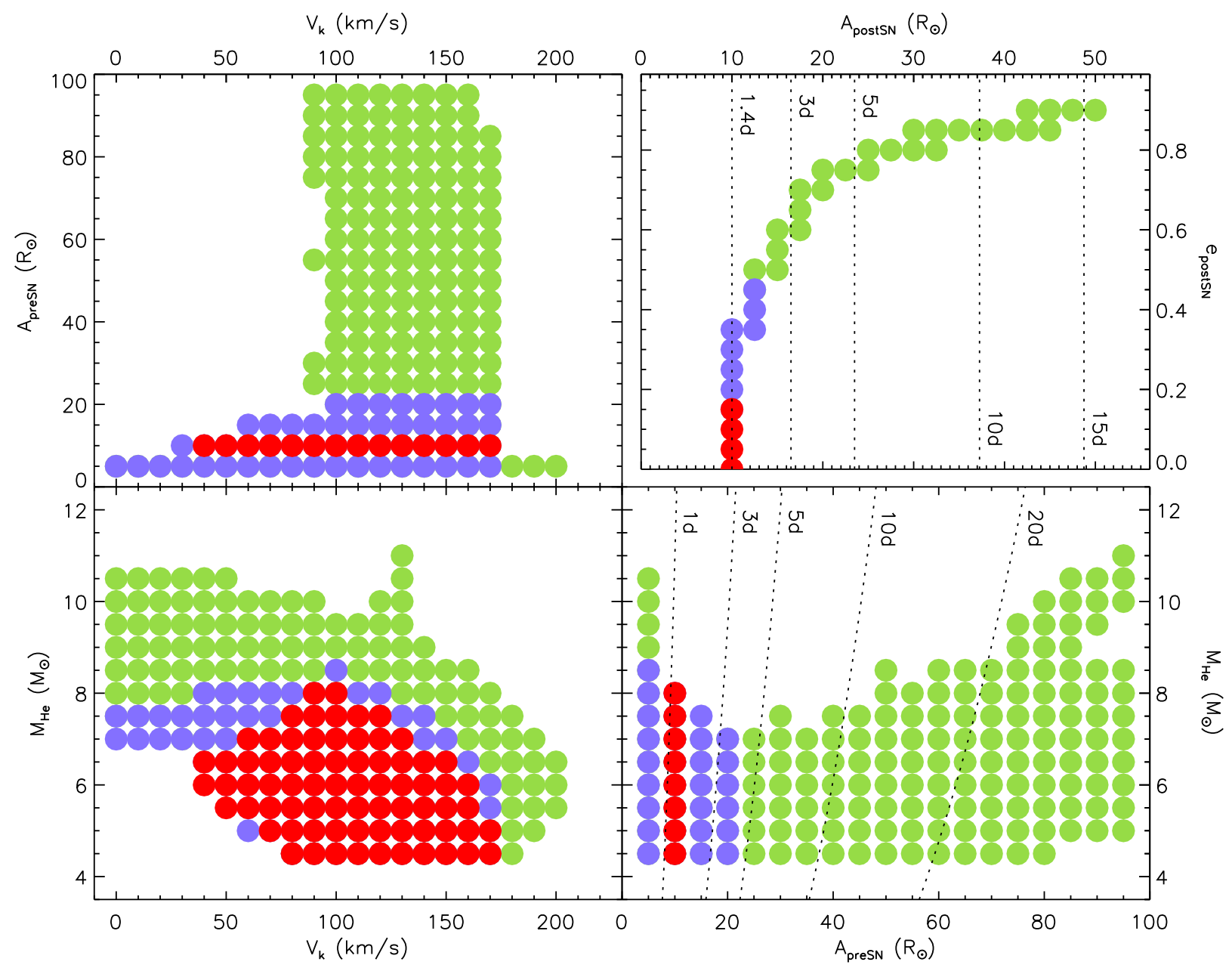

Fig. 6.- Constraints on the pre-SN helium star mass $M_{\mathrm{He}}$, the magnitude of the kick velocity $V_{k}$, the pre-SN orbital separation $A_{\text {preSN }}$, and the post-SN orbital separation $A_{\text {postSN }}$ and eccentricity $e_{\text {postSN }}$ associated with the successful MT sequence BP62 (see Table 3). Light grey circles (green in the electronic edition) correspond to progenitors for which RLO at periastron occurs more than 200 Myr earlier than dictated by the initial age of the donor star in the MT sequence, dark grey circles (blue in the electronic edition) correspond to progenitors for which RLO at periastron occurs between 50 and 200 Myr earlier, and black circles (red in the electronic edition) correspond to progenitors for which RLO at periastron occurs within $50 \mathrm{Myr}$ of the time dictated by the MT sequence. The black circles (red in the electronic edition) correspond to our preferred set of solutions. The dotted lines in the right-hand panels are lines of constant orbital period. 
To illustrate the derivation of the constraints, we consider the conservative MT sequence BP62 (see Table 3) with initial BH mass $M_{\mathrm{BH}}=4.5 M_{\odot}$, initial donor mass $M_{2}=2.5 M_{\odot}$, and initial orbital period $P_{\text {orb }}=1.4$ days (note that this is the same sequence as used in Fig. 5). The constraints obtained for the mass $M_{\mathrm{He}}$ of the BH's helium star progenitor, the magnitude $V_{k}$ of the kick velocity imparted to the BH at birth, the pre-SN orbital separation $A_{\text {preSN }}$, and the post-SN orbital separation $A_{\text {postSN }}$ and eccentricity $e_{\text {postSN }}$ are presented in Fig. 6. The tight correlation between $A_{\text {postSN }}$ and $e_{\text {postSN }}$ (top tight panel) arises from matching the post-SN orbital elements to those of the MT sequence at the onset of RLO. As discussed in Section 6 , pairs of $A_{\text {postSN }}$ and $e_{\text {postSN }}$ are considered to be acceptable if, after following the orbital evolution for a time equal to the age of the donor at RLO onset, the total angular momentum is equal to that given by the MT sequence within the uncertainties of the numerical calculations. This condition, however, does not guarantee that the eccentric orbit can accommodate the $\mathrm{BH}$ companion throughout the time interval between $\mathrm{BH}$ formation to RLO. In particular, for significantly eccentric orbits, RLO at periastron may occur at a time when the donor star has not yet reached the right evolutionary stage (see the bottom right panel in Fig. 5 and the associated discussion). These pairs of $\left(A_{\text {postSN }}, e_{\text {postSN }}\right)$ will therefore be valid post-SN orbital parameters only if RLO at periastron does not significantly affect the star and the orbit. In view of our limited knowledge of MT in eccentric binaries and its effects on the orbital elements, we include these solutions in the presentation of the progenitor constraints, although we consider them to be less compatible with the initial RLO conditions imposed by the MT sequence. In order to distinguish between solutions that lead to MT at periastron at times significantly close to RLO onset in the MT sequence and those that do not, we separate them into three groups in Fig. 6: solutions for which RLO at periastron occurs much too early (i.e. more than $200 \mathrm{Myr}$ ) are represented by light grey circles (green in the electronic edition), solutions for which RLO at periastron occurs between 50 and 200 Myr too early are represented by dark grey circles (blue in the electronic edition), and solutions for which RLO at periastron occurs within $50 \mathrm{Myr}$ of the proper time given by the MT sequence are represented by black circles (red in the electronic edition). We consider the progenitor constraints associated with the latter solutions to be the most compatible with the MT sequence under consideration and therefore refer to them as our "preferred set" of solutions.

The constraints on $A_{\text {preSN }}, A_{\text {postSN }}$, and $e_{\text {postSN }}$ are very tight when restricted to progenitors for which RLO at periastron occurs within $\simeq 50 \mathrm{Myr}$ of the donor age imposed by the MT sequence: $A_{\text {preSN }} \simeq 10 R_{\odot}, A_{\text {postSN }} \simeq 10 R_{\odot}$, and $e_{\text {postSN }} \lesssim 0.15$. These ranges broaden somewhat when solutions for which RLO at periastron occurs between $\simeq 50$ and $\simeq 200$ Myr too early are included, but overall the differences with our preferred set of solutions is not too large. When RLO at periastron occurs more than $\simeq 200$ Myr too early, 
additional highly eccentric post-SN orbits with large semi-major axes become available. Correspondingly, the constraints on the pre-SN orbital separation also extend to very large values. A similar behavior is observed for the constraints on $M_{\mathrm{He}}$ and $V_{k}$. When RLO at periastron occurs within $\simeq 50 \mathrm{Myr}$ of the required donor age, $4.5 M_{\odot} \lesssim M_{\mathrm{He}} \lesssim 8.0 M_{\odot}$ and $40 \mathrm{~km} \mathrm{~s}^{-1} \lesssim V_{k} \lesssim 170 \mathrm{~km} \mathrm{~s}^{-1}$. For our preferred set of solutions, the formation of the $\mathrm{BH}$ must therefore be accompanied by a natal kick. When solutions for which RLO at periastron occurs within $\simeq 50-200 \mathrm{Myr}$ of the required donor age are included, the ranges slightly broaden to $4.5 M_{\odot} \lesssim M_{\mathrm{He}} \lesssim 8.5 M_{\odot}$ and $V_{k} \lesssim 170 \mathrm{~km} \mathrm{~s}^{-1}$. Hence, the derived constraints are fairly robust even when we allow RLO at periastron to occur up to $\simeq 200$ Myr too early. Somewhat larger variations are found when RLO at periastron occurs more than $\simeq 200 \mathrm{Myr}$ too early, but MT for these systems is likely to significantly affect both the star and the orbit so that the final parameters after $\simeq 480 \mathrm{Myr}$ (the required age of the donor at the start of RLO) of orbital evolution are probably incompatible with the initial conditions of the considered MT sequence and thus these solutions are not favored. Since highly eccentric post-SN orbital configurations always fall under the latter category, we do not consider post-SN orbital eccentricities larger than 0.9 in the derivation of any of the constraints.

To understand the core-collapse event leading to the formation of the $\mathrm{BH}$, we are mainly interested in the constraints derived for the mass of the BH's helium star progenitor and the kick velocity that may have been imparted to the $\mathrm{BH}$ at birth. For the remainder of this section, we therefore restrict ourselves to presenting the constraints derived for these two quantities. Given that there is no way to distinguish which of the various successful MT sequences corresponds to the true progenitor of GROJ1655-40, we derive the constraints for each successful sequence and examine them collectively. The constraints resulting from our preferred set of solutions for each individual sequence are summarized in the last two columns of Tables 2-3. 


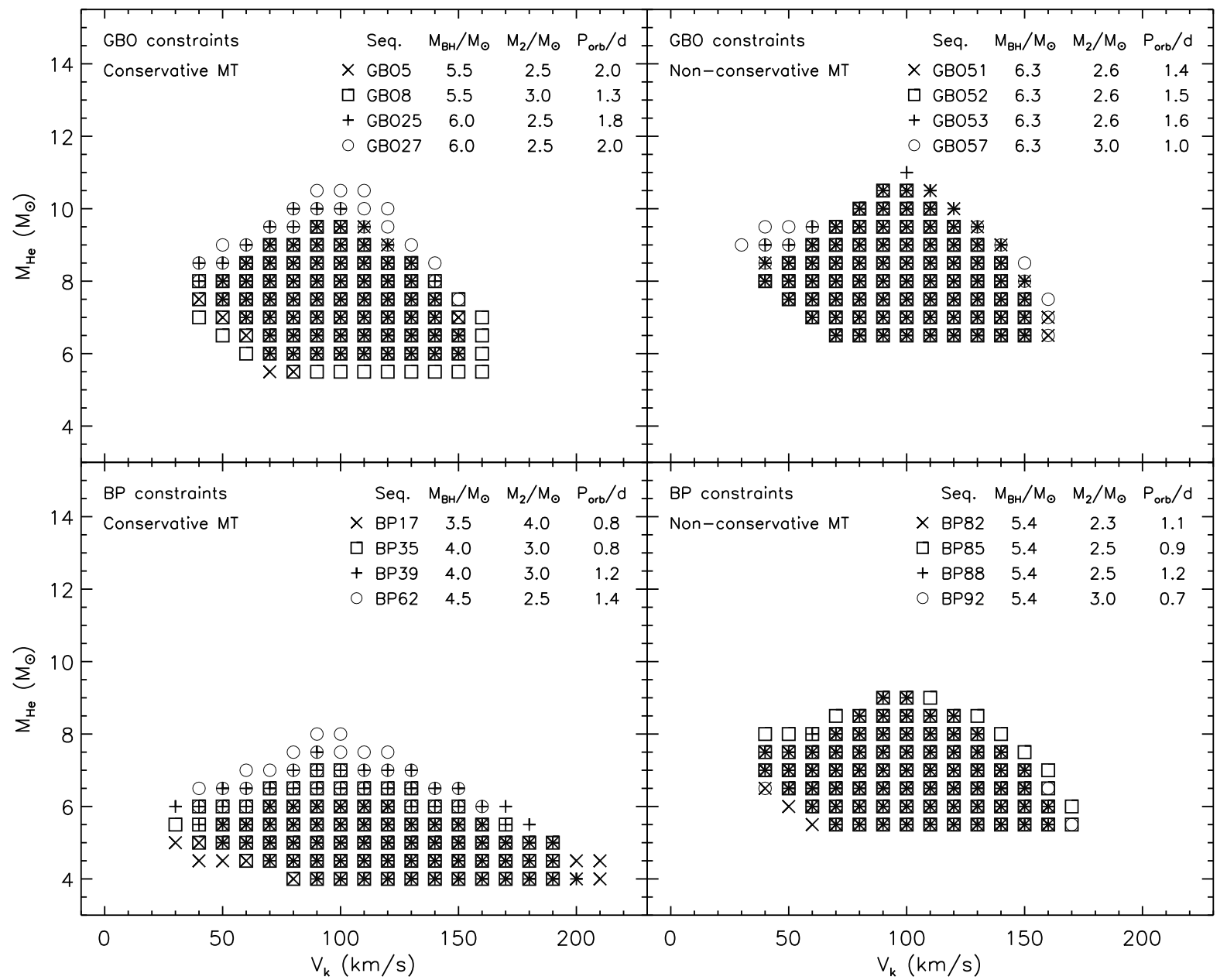

Fig. 7.- Constraints on the mass $M_{\mathrm{He}}$ of the BH's helium star progenitor and the magnitude $V_{k}$ of the kick velocity imparted to the BH for some selected successful MT sequences (see Tables 2-3 for details). For clarity, only solutions for which RLO at periastron occurs within $50 \mathrm{Myr}$ of the time imposed by the MT sequence are represented. 
In Fig. 7, we first show the constraints on $M_{\mathrm{He}}$ and $V_{k}$ for a selection of MT sequences with initial parameters on the edges of the regions leading to successful sequences (see Fig. 2) for conservative and non-conservative MT and for GBO and BP constraints separately. For clarity, only our preferred sets of solutions are plotted. The variations in the binary properties at the onset of RLO are seen to yield only small variations in the constraints for $M_{\mathrm{He}}$ and $V_{k}$. We can therefore be confident that the derived constraints are not too much affected by the finite number of successful MT sequences found, as long as we bare in mind that we outlined the ranges of initial component masses and orbital periods at the onset of RLO leading to successful sequences to within a predetermined uncertainty of $0.5 M_{\odot}$ in the masses and 0.1 days in the orbital period. 


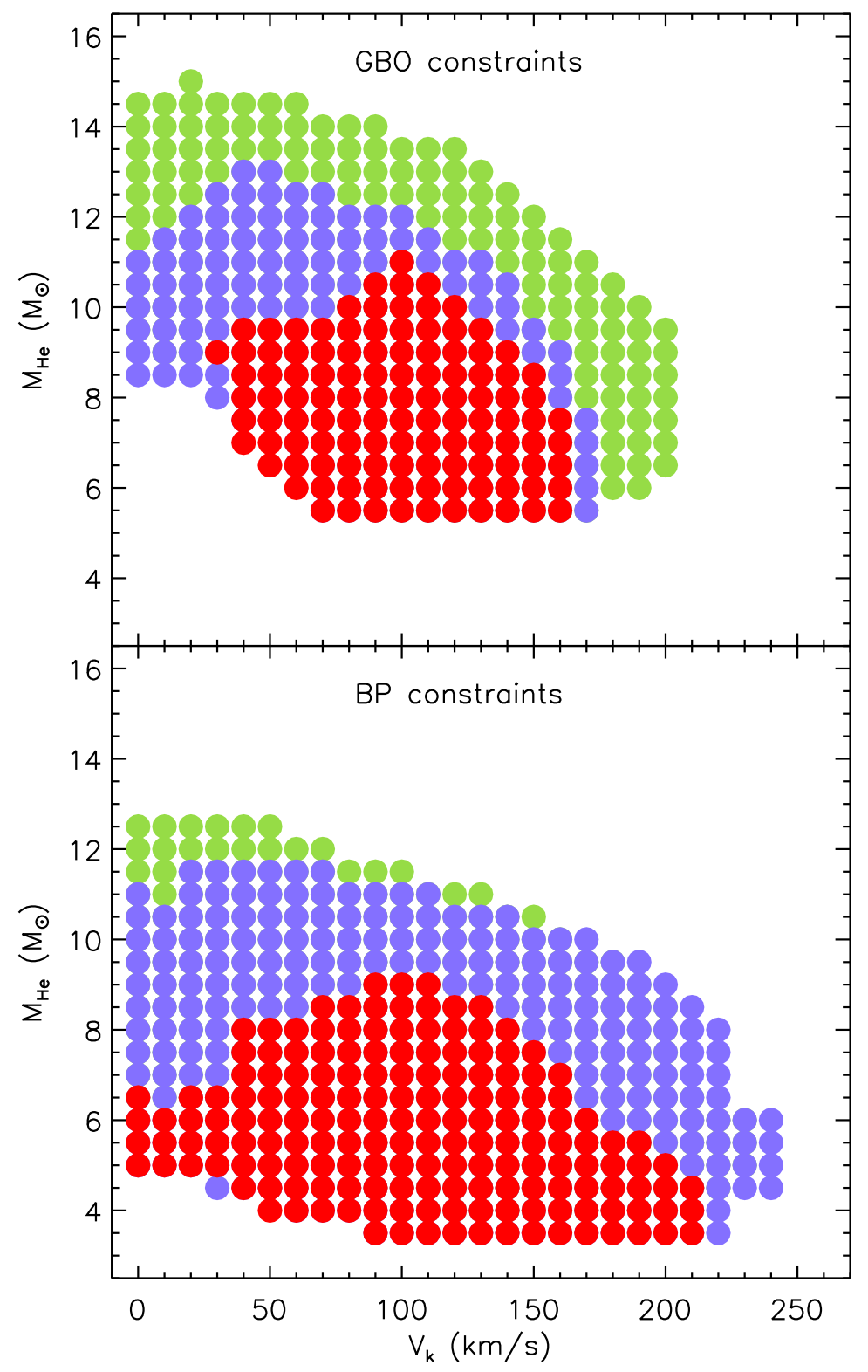

Fig. 8.- Constraints on the mass $M_{\mathrm{He}}$ of the BH's helium star progenitor and the magnitude $V_{k}$ of the kick velocity that may have been imparted to the $\mathrm{BH}$ obtained by combining the results from all successful MT sequences (see Tables 2-3 for sequence details). The different colors have the same meaning as in Fig. 6. 
Since we expect the true MT sequence describing the evolution of GRO J1655-40 from the start of the X-ray phase to its present configuration to be encompassed by the successful MT sequences, we overlay the $M_{\mathrm{He}}$ and $V_{k}$ constraints for all the successful conservative and non-conservative MT sequences found for GBO and BP system parameters in Fig. 8. It follows that even the combination of the extreme cases of fully conservative and fully nonconservative MT does not significantly relax the constraints on the progenitor masses and kick velocities with respect those obtained for individual sequences. For our preferred set of solutions, the helium star mass and kick velocity are constrained to $5.5 M_{\odot} \lesssim M_{\mathrm{He}} \lesssim 11.0 M_{\odot}$ and $30 \mathrm{~km} \mathrm{~s}^{-1} \lesssim V_{k} \lesssim 160 \mathrm{~km} \mathrm{~s}^{-1}$ in the case of GBO parameters, and to $3.5 M_{\odot} \lesssim M_{\mathrm{He}} \lesssim$ $9.0 M_{\odot}$ and $0 \mathrm{~km} \mathrm{~s}^{-1} \lesssim V_{k} \lesssim 210 \mathrm{~km} \mathrm{~s}^{-1}$ in the case of BP parameters ${ }^{4}$. On average, higher progenitor masses furthermore tend to be associated with lower kick velocities since more mass loss requires less of kick to achieve the same result. A similar correlation was found by Willems et al. (2004) for the progenitor of PSR J0737-3039B. Overlaying our preferred set of solutions for the other binary parameters yields $5 R_{\odot} \lesssim A_{\text {preSN }} \lesssim 15 R_{\odot}, 10 R_{\odot} \lesssim$ $A_{\text {postSN }} \lesssim 15 R_{\odot}$, and $e_{\text {postSN }} \lesssim 0.3$ for GBO system parameters, and $5 R_{\odot} \lesssim A_{\text {preSN }} \lesssim 15 R_{\odot}$, $7 R_{\odot} \lesssim A_{\text {postSN }} \lesssim 10 R_{\odot}$, and $e_{\text {postSN }} \lesssim 0.35$ for BP system parameters.

\footnotetext{
${ }^{4}$ It is interesting to note that, for our preferred set of solutions, the symmetric SN solution in the case of BP system parameters imparts a kick of $\simeq 50-70 \mathrm{~km} \mathrm{~s}^{-1}$ to the binary's center of mass. If all solutions associated with $V_{k}=0 \mathrm{~km} \mathrm{~s}^{-1}$ are considered, the mass loss imparts a kick of $\simeq 45-115 \mathrm{~km} \mathrm{~s}^{-1}$ to the binary's center of mass, corresponding to the entire range of admissible post-SN peculiar velocities found from the Galactic motion calculations presented in Section 5.
} 


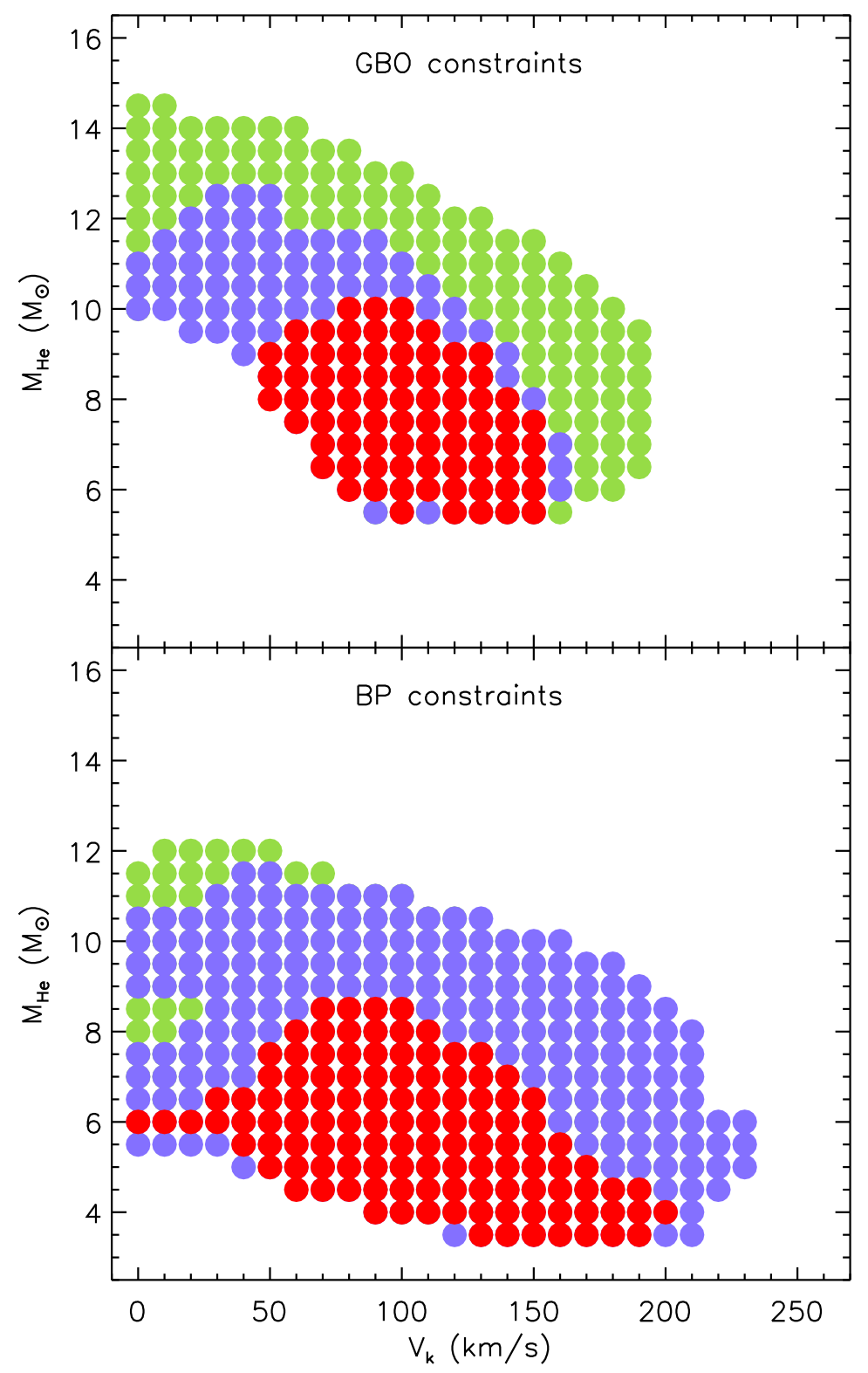

Fig. 9.- As Fig. 8, but for a fixed error of $\pm 10 \mathrm{~km} \mathrm{~s}^{-1}$ on the post-SN peculiar velocity. 
As mentioned in Section 5, the errors on the post-SN peculiar velocity, which imposes stringent constraints on $M_{\mathrm{He}}$ and $V_{k}$, were estimated by considering the Galactic orbits of GRO J1655-40 for all possible combinations of the lower and upper limits on the observed distance and velocity components. Since this likely overestimates the true errors on the post-SN peculiar velocity, we reconsidered the derivation of the $\mathrm{BH}$ progenitor mass and kick velocity constraints for a fixed error of $\pm 10 \mathrm{~km} \mathrm{~s}^{-1}$ on $V_{\text {pec,postSN }}$ (represented by the dark grey area in Fig. 4). The resulting constraints are shown in Fig. 9. For a given helium star mass, the range of possible kick velocities generally narrows with respect to the range found in Fig. 8, although the overall ranges in $M_{\mathrm{He}}$ and $V_{k}$ do not differ significantly. Are results are therefore remarkably robust with respect to the uncertainties in the post-SN peculiar velocity.

We conclude that in the case of GBO parameters, it is likely that the BH in GRO J165540 received a kick at birth. Depending on the errors in the post-SN peculiar velocity, the minimum kick velocity is $\simeq 30-50 \mathrm{~km} \mathrm{~s}^{-1}$. In the case of BP parameters, a kick is much less of a requirement unless very small errors on the post-SN peculiar velocity are assumed. The main differences between the constraints derived for GBO and BP parameters are related to the higher initial BH masses and the correspondingly higher helium star masses which reduce the center-of-mass velocity imparted to the binary by a symmetric SN explosion in the case of GBO parameters.

\section{CORE-COLLAPSE HYDRODYNAMIC SIMULATIONS AND CONSTRAINTS}

To model the collapse of the possible BH progenitors of GRO J1655-40, we use the pre-collapse stellar models of Rauscher et al. (2003). Since the binary formed in the galactic disk, we focus on the solar metallicity models. We assume the MT phase that revealed the helium-star occurred late enough in the star's life ( case C MT) that its structure was only slightly modified due to the loss of the hydrogen envelope, allowing us to simply remove the hydrogen envelopes from these single-star models to incorporate the effects of close binary evolution. One of the major uncertainties in our calculations is the mass loss. The high mass-loss rates in current stellar models are often too high to produce BHs in close binaries. This excessive mass-loss is especially extreme in the Wolf-Rayet phase, effectively making it impossible to make a $\mathrm{BH}$ from a star that loses its hydrogen envelope before the end of helium burning (Wellstein \& Langer 1999).

With current stellar models, we can not make BHs from systems that lose their hydrogen envelopes in a Case B MT phase. Even limiting ourselves to Case C MT, it is difficult to 
produce helium cores above $11-12 M_{\odot}$. This is the first constraint that stellar and corecollapse models can place on the helium star progenitor: If wind mass-loss rates are correct, $M_{\mathrm{He}} \lesssim 11-12 M_{\odot}$. This may be a real constraint, but could also be due to over-estimates of the mass-loss rates from winds by the stellar evolution community. To mimic helium-star BH progenitors of high mass up to $15 M_{\odot}$, we use the $10^{-4}$ solar metallicity progenitors from Rauscher et al. (2003). Since the metallicity principally effects the mass loss alone (Heger et al. 2003), these models can be used to represent the fate of solar-metallicity stars with a reduced mass loss.

These stellar models/profiles are mapped into a 1-dimensional Lagrangian hydrodynamics code (Herant et al. 1994; Fryer et al. 1999). The equation of state used in the simulations includes a nuclear equation of state at high densities (Lattimer \& Swesty 1991), and below $5 \times 10^{11} \mathrm{gcm}^{-3}$ an equation of state for nuclei obeying Boltzmann statistics (Blinnikov, Dunina-Barkovskaya, \& Nadyozhin 1996, Timmes \& Arnett 1999). Both in the high density equation regime and in the low-density regime above temperatures of $5 \times 10^{9} \mathrm{~K}$, abundances are determined assuming nuclear statistical equilibrium. Between $2 \times 10^{8}$ and $5 \times 10^{9}$, the abundances are evolved using a 14-element nuclear network (Benz, Hills, \& Thielemann 1989) which yields reasonable approximations for the nuclear energy generation rate (Timmes, Hoffman \& Woosley 2000). 
Table 4. Core-Collapse Models

\begin{tabular}{lcccccc}
\hline \hline $\begin{array}{c}\text { Progenitor } \\
\text { Mass }\left(M_{\odot}\right)\end{array}$ & $f_{\nu}{ }^{\mathrm{a}}$ & $\begin{array}{c}\text { Remnant } \\
\text { Mass }\left(M_{\odot}\right)\end{array}$ & $\begin{array}{c}\text { Energy } 3000 \mathrm{~km} \\
\left(10^{51} \mathrm{erg}\right)\end{array}$ & $\begin{array}{c}\text { K.E.breakout } \\
\left(10^{51} \mathrm{erg}\right)\end{array}$ & $\begin{array}{c}\mathrm{t}_{\text {remnant }} \\
10^{6} \mathrm{yr}\end{array}$ & $\begin{array}{c}\mathrm{V}_{\text {remnant }}{ }^{\mathrm{c}} \\
\mathrm{V}_{51}\end{array}$ \\
\hline 6.2 & 0.9 & 6.2 & 0.0 & 0. & 0. & 0. \\
6.2 & 1 & 4.59 & 0.9 & 0.068 & 1.0 & 0.08 \\
6.2 & 1.1 & 2.39 & 1.4 & 0.19 & 1.4 & 0.2 \\
8.2 & 1.0 & 6.33 & 0.8 & 0.083 & 1.0 & 0.09 \\
8.2 & 1.1 & 5.42 & 1.0 & 0.17 & 1.3 & 0.18 \\
8.2 & 1.2 & 3.16 & 1.6 & 0.32 & 1.6 & 0.33 \\
10.0 & 1.0 & 7.14 & 0.8 & 0.16 & 1.3 & 0.17 \\
10.0 & 1.06 & 4.16 & 1.6 & 0.64 & 2.0 & 0.65 \\
10.0 & 1.12 & 2.26 & 2.0 & 1.3 & 2.5 & 1.3 \\
14.9 & 0.92 & 14.9 & 0. & 0. & 0. & 0. \\
14.9 & 1.00 & 5.34 & 7.1 & 2.26 & 3.0 & 2.2 \\
14.9 & 1.04 & 2.23 & 12.0 & 7.7 & 4.4 & 7.1 \\
\hline
\end{tabular}

a $f_{\nu}=1$ corresponds to the lowest factor used that still produces an explosion. All other values are relative to this quantity.

${ }^{\mathrm{b}}$ Kinetic energy

${ }^{c}$ The effective volume covered by the SN remnant before it merges with the interstellar medium as a fraction of the volume encompassed by a $10^{51} \mathrm{erg}$ explosion. 
Neutrino transport is mediated using an explicit flux-limited algorithm (Herant et al. 1994). Above a radius determined by the neutrino mean free path, the neutrinos are transported in the free streaming limit, escaping the star instantly. For most models (e.g., Fryer et al. 1999), this radius is chosen such that less than 3-5\% of the neutrinos in the free streaming limit interact with the matter as it leaves the star. In the models presented here, the neutrinos in this free streaming limit are increased by a factor $f_{\nu}$ and the limit is set to force roughly $10 \%$ of the neutrinos in the free-streaming limit to interact with matter. This effectively raises the neutrino energy in the "gain" region where neutrino heating deposits energy into the stellar material. By increasing $f_{\nu}$, we increase the neutrino heating and can drive a SN explosion (Table 4 lists the suite of these simulations).

The high sensitivity of remnant mass on our fudge factor $f_{\nu}$ demonstrates just how delicate the balance between success and failure is for the neutrino-driven mechanism. This delicate balance is one reason why core-collapse theorists have struggled to get consistent explosions in their simulations of these explosions (getting all of the necessary physics accurate to the $10 \%$ level is a daunting task). Although a robust mechanism may actually exist where $10 \%$ effects are not important, it is important to understand that the actual progenitors of core-collapse (massive stars with masses above $8-9 M_{\odot}$ ) are not too dissimilar, yet nature produces a range of remnant masses. It appears that nature too is sensitive to small changes in the details.

A massive star collapses until it reaches nuclear densities. A proto-NS is formed and the explosion occurs if the neutrinos from the cooling NS can provide enough energy to drive off the rest of the star. As the explosion is launched, matter expands off the NS surface, and the cells are split to retain resolution with our Lagrangian code near the NS surface. After $\sim 1 \mathrm{~s}$ the explosion shock is well-developed $(\sim 3000 \mathrm{~km})$. At this point, the NS is cut out of the simulation and modelled as a gravitational source with a hard boundary. The explosion is then followed until the shock reaches the edge of the surface of the helium star. At this point, the energy in the exploding star is primarily in kinetic energy and we can determine the rough SN explosion energy of these objects (see Table 4). We also determine the BH mass by assuming all material with velocities less than the escape velocity will ultimately fall back onto the NS (see Table 4). For all cases, we can produce a BH with a mass lying somewhere between $\sim 4.0-6.5 M_{\odot}$, but the resulting energies for these BHs lies between $\sim 0-2 \times 10^{51} \mathrm{erg}$.

Our understanding of core-collapse theory can also be used to constrain the BH progenitors. Fryer (1999) found that, using a multi-dimensional core-collapse code, massive stars above $\sim 20 M_{\odot}$ achieved explosion energy (when the shock was near 1000-3000 km) of roughly $0.6 \times 10^{51} \mathrm{erg}$. There are a number of uncertainties in these "first-principle" collapse 
calculations and this answer can change considerably with more accurate codes. But if we take these simulations at face value and assume that the energy is, within a factor of 2 , accurate, we can also rule out some progenitors. From Table 4, we see that only progenitors below $10 M_{\odot}$ can obtain the appropriate $\mathrm{BH}$ mass with less than about $1.2 \times 10^{51} \mathrm{erg}$ of explosion energy when the shock is launched. This constraint (the second from stellar and core-collapse models) is strongest for small BH masses. 


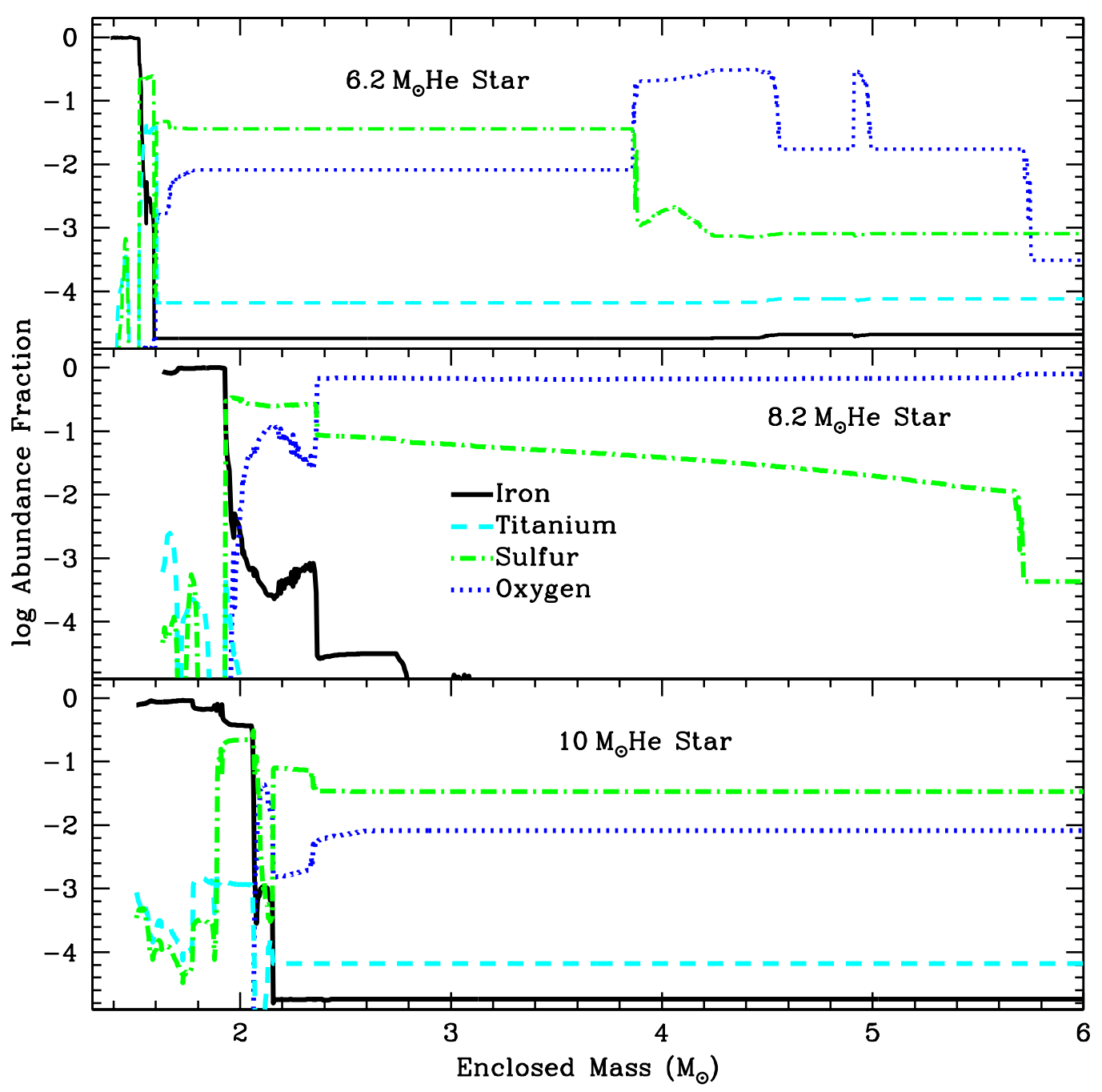

Fig. 10.- Abundance fractions of iron (solid line), titanium (dashed line), sulfur (dot-dashed line), and oxygen (dotted line) versus enclosed mass for three models: $M_{\mathrm{He}}=6.2 M_{\odot}, f_{\nu}=1$ (top), $M_{\mathrm{He}}=8.2 M_{\odot}, f_{\nu}=1.1$ (middle), and $M_{\mathrm{He}}=10.0 M_{\odot}, f_{\nu}=1.06$ (bottom). In these explosions, roughly the inner $4-5 M_{\odot}$ of material will fall back onto the remnant, forming a BH. Without mixing, none of the explosions will produce iron or titanium far enough out to enrich the companion. Indeed, using estimates from Hungerford et al. (2003), even the mixing that occurs in symmetric 3-dimensional simulations is not sufficient to lead to iron and titanium enrichment of the companion. Only asymmetric explosions can produce enough mixing. The level of asymmetry required depends primarily on how far out the iron and titanium are formed. Notice that the iron and titanium is formed nearly twice as far out in mass coordinate for the $10 M_{\odot}$ progenitor compared to the $6.2 M_{\odot}$ progenitor. The level of asymmetry to produce the necessary enrichment is much higher for the $6.2 M_{\odot}$ progenitor when compared to the $10 M_{\odot}$ progenitor. 
We can also use the nucleosynthetic yields to constrain the models. As noted above, the 14 isotope nuclear network used in the hydrodynamics simulations is used only to determine energetics. To obtain more detailed abundance information, we post-processed the thermodynamic trajectories of our Lagrangian particles with a modern 500 isotope network (e.g., Timmes 1999). For simplicity we assumed an initial electron fraction of 0.5. The iron, titanium, sulfur and oxygen yields as a function of enclosed mass for three representative models are presented in Fig. 10. These three models: $M_{\mathrm{He}}=6.2 M_{\odot}, f_{\nu}=1, M_{\mathrm{He}}=8.2 M_{\odot}$, $f_{\nu}=1.1, M_{\mathrm{He}}=10 M_{\odot}, f_{\nu}=1.06$, each produce BHs with masses above $4 M_{\odot}$. Oxygen and even some sulfur is formed well out in the star, but titanium and iron are formed in the most intense burning at the base of the explosion. However, note that the more massive stars produce iron and titanium further out than the low-mass stars. This occurs because the more massive stars require stronger explosions to produce BHs of comparable mass to the lower mass stars. The stronger explosions produce intensive nuclear burning further out in the star, ultimately producing iron further out in the star as well.

Do such abundance profiles provide additional, useful information? We often use the nucleosynthetic signature from supernovae to constrain SN progenitors. However, the yields from these stars must mix out extensively in the explosion just to get ejected. These $\mathrm{BH}$ forming stars will not dominate the nuclear yields of the Galaxy. In addition, due to their weak explosions, the nucleosynthetic yields from these explosions are not likely to mix extensively into the interstellar medium. The timescale $\left(t_{\max }\right)$ for the SN shock to once again become part of the interstellar medium (when the shock velocity drops below the sound speed of the ambient medium) is roughly (McKee \& Hollenbach 1980):

$$
t_{\max }=2.3 \times 10^{6} E_{51}^{0.32} c_{\mathrm{s} 6}^{-1.45} n_{0}^{-0.36} \mathrm{yr},
$$

where $E_{51}$ is the explosion energy in units of $10^{51} \mathrm{erg}$; and $c_{\mathrm{s} 6} \approx 1$ in units of $10^{6} \mathrm{~cm} \mathrm{~s}^{-1}$ and $n_{0} \approx \mathrm{pc}^{-3}$ are, respectively, the sound speed and particle density of the ambient medium. The corresponding maximum extent $\left(r_{\max }\right)$ of the SN shock is:

$$
r_{\max }=76 E_{51}^{0.32} c_{\mathrm{s} 6}^{-0.45} n_{0}^{-0.36} \mathrm{pc} .
$$

The timescales and effective volumes (as a fraction of a normal $10^{51}$ erg explosion) of the remnants of these BHs are given in Table 4. With such short-lived, small remnants, it is unlikely that we will be able to constrain these stars by Galactic or remnant abundances.

However, Israelian et al. (1999) observed the companion of GRO J1655-40 during a quiescent X-ray phase using the high-resolution spectrograph at Keck. At visible wavelengths, they found that the abundances of $\mathrm{O}, \mathrm{Mg}, \mathrm{S}, \mathrm{Si}$, and $\mathrm{Ti}$ were 6-10 times larger than solar. They also found that $[\mathrm{Fe} / \mathrm{H}]=0.1 \pm 0.2$, i.e. almost solar. The most straightforward and logical way of explaining these overabundances, and the one proposed by Israelian et al., 
is that these elements were produced during explosive oxygen and silicon burning during the $\mathrm{BH}$ formation and have enriched the companion. But to cause this enrichment, these elements must make it to the companion star. For the iron and titanium, this can be quite a difficult task. At the time of explosion the companion was roughly $5-15 R_{\odot}$ from the center of the collapsing star. But our 1-dimensional explosion models find that the iron and titanium does not make it beyond $0.05 R_{\odot}$ before falling back onto the compact remnant. Even in 3-dimensional simulations, the amount of mixing found in symmetric models does not place the iron or titanium beyond $0.5 R_{\odot}$ before these elements fall back onto the compact remnant. The extensive mixing required to eject these elements requires large asymmetries in the explosion (Hungerford et al. 2003; Hungerford et al. 2004). Hence, if the enrichment of the companion of GRO J1655-40 is arising from the explosion forming the BH, its SN must have been asymmetric! The amount of asymmetry reaches a minimum with the lower mass progenitors (especially with massive BHs). For example, in the extreme case of a $\mathrm{BH}$ mass of $6.2 M_{\odot}$, the $6.2 M_{\odot}$ progenitor will not, no matter what the asymmetry, be able to enrich the companion. Even with smaller BH masses, it is likely that the helium progenitor for this system is more massive $\left(\gtrsim 8 M_{\odot}\right)$. This is our third, and final, constraint on the helium star mass.

In principal, the exact abundances of the companion star could be used to determine the best-fitting BH progenitor (see Podsiadlowski et al. 2002b). However, the uncertainties in the level of asymmetry and the uncertainties of the effects of asymmetry on the actual nuclear yield make it difficult to produce any accurate quantitative results. These uncertainties must then be folded into uncertainties in the accretion onto the companion. The study of asymmetry-induced mixing is still in its infancy. When it is better understood, the actual composition of the companion star can be used to constrain the progenitors as well.

Although there exist a number of uncertainties in stellar evolution and core-collapse calculations, these models can place additional constraints on the BH progenitor for GRO J165540:

- I: $M_{\mathrm{He}} \lesssim 11-12 \mathrm{M}_{\odot}$ - Stellar Winds. With current mass-loss rates for winds, helium cores above this mass are simply not produced. The limitation of this constraint lies in the uncertainties in stellar winds. Given that BH masses for some other systems have been estimated to be as high as $18 M_{\odot}$ (McClintock \& Remillard 2004, and references therein), we do not consider this constraint to be very stringent.

- II: $M_{\mathrm{He}} \lesssim 10 \mathrm{M}_{\odot}$ - Supernova Energetics. If explosion energies for these massive stars do decrease with increasing stellar mass, it is impossible to get low-mass BHs with more massive helium stars. This constraint is strongest for the low-end of the BH 
mass range allowed for GRO J1655-40. This constraint is limited by uncertainties in the core-collapse mechanism.

- III: $M_{\mathrm{He}} \gtrsim 8 \mathrm{M}_{\odot}$ - Nucleosynthetic Yields. If the high abundances of the companion to GRO J1655-40 are due to ejecta enrichment, large asymmetries are required to mix out the iron and titanium so that it is ejected. Prohibitively large asymmetries are required for low-mass progenitors. This constraint is strongest for BH masses at the high-end of the allowed range for GRO J1655-40. The dominant limitation in this constraint arises from the uncertainty of the true enrichment in the companion of GRO J1655-40.

In addition to constraints on the progenitor mass, our calculations also show how observations of GRO J1655-40 can constrain SN explosions. If the anomalous abundances in the companion to GRO J1655-40 truly arise from enrichment from the BH-forming SN, then this object provides some of the best evidence not only for the occurrence of a SN explosion, but also for asymmetries in the explosions (Israelian et al. 1999, Podsiadlowski et al. 2002b). A better constraint on the progenitor (as well as a better understanding of structure of the asymmetries) could significantly constrain the magnitude of the asymmetry in this explosion.

\section{DISCUSSION AND CONCLUSIONS}

In this paper, we initiated a comprehensive study on the formation and evolution of Galactic XRBs. Based on the current binary properties and the current position and motion of observed XRBs, the kinematic and evolutionary history is traced back in time and constraints are derived for the pre-SN binary progenitor and for the kick velocity that may have been imparted to the compact object at birth. The analysis consists of the following elements: (i) MT calculations to model the ongoing X-ray phase, (ii) orbital evolution due to tides and gravitational radiation between the compact object formation time and the onset of RLO, (iii) motion of the system in the Galaxy after the formation of the compact object, (iv) binary orbital dynamics at the time of core collapse, and (v) hydrodynamic modeling of the actual core collapse event.

As a first application, we constrained the progenitor properties and the formation of the BH in the soft X-ray transient GRO J1655-40. For this purpose, we calculated a large number of MT sequences to map the currently observed binary properties (component masses, position of the donor in the H-R diagram, orbital period, and transient behavior) to those at the onset of the MT phase. Uncertainties related to the accretion of matter by the BH are incorporated by considering both conservative (for sub-Eddington rates) and non-conservative 
MT. For the current system configuration, we considered both the parameters derived by GBO and those derived by BP (see Table 1 for details). In the case of GBO parameters, successful MT sequences able to reproduce all currently observed system properties were found for initial $\mathrm{BH}$ masses $M_{\mathrm{BH}} \simeq 5.5-6.3 M_{\odot}$, initial donor star masses $M_{2} \simeq 2.5-3.0 M_{\odot}$, and initial orbital periods $P_{\text {orb }} \simeq 1.0-2.0$ days. In the case of $\mathrm{BP}$ parameters, successful sequences were found for initial $\mathrm{BH}$ masses $M_{\mathrm{BH}} \simeq 3.5-5.4 M_{\odot}$, initial donor star masses $M_{2} \simeq 2.3-4.0 M_{\odot}$, and initial orbital periods $P_{\text {orb }} \simeq 0.7-1.5$ days. For both cases, all successful sequences have a MS donor star at the onset of RLO. A graphical representation of the initial donor masses and orbital periods leading to successful MT sequences is presented in Fig. 2. Properties of the sequences are given in Tables 2-3.

The results of the MT calculations are in good agreement with previous investigations. Regős, Tout, \& Wickramasinghe (1998) showed that the present-day properties of GRO J1655-40 (as determined by Orosz \& Bailyn 1997) could be reproduced by a binary initially consisting of a $2.75 M_{\odot}$ MS star orbiting a $6.6 M_{\odot} \mathrm{BH}$ with a period of 1.935 days. These initial parameters are fairly close to those of the successful MT sequence GBO26 in Table 2, except for the higher initial BH mass adopted by Regös et al. (1998). This small difference can be attributed to the higher current BH mass of $7.02 \pm 0.22 M_{\odot}$ estimated by Orosz \& Bailyn (1997) as well as to some different input physics in the stellar evolution codes, such as, e.g., the amount of convective overshooting.

Kolb et al. (1997) and Kolb (1998), on the other hand, suggested that GRO J1655-40 may be in a very short-lived evolutionary phase in the Hertzsprung gap during which the expansion of the donor's radius temporarily halts or even reverses. As a consequence, the MT rate drops below the high rates typically expected for Hertzsprung-gap donor stars, allowing for a short phase of transient behavior. In view of the small size of the relevant region in the Hertzsprung gap (see, e.g., Fig. 2 in Kolb et al. 1997) and the associated short evolutionary time scales, progenitors which start RLO while the donor is still on the MS seem statistically more favorable. We therefore did not attempt to reproduce the small transient strip in the Hertzsprung gap found by Kolb et al. (1997) and Kolb (1998). In their investigation, Kolb et al. (1997) also adopted the BH mass derived by Orosz \& Bailyn (1997), which is lower than that derived by both $\mathrm{GBO}\left(M_{\mathrm{BH}}=6.3 \pm 0.5 M_{\odot}\right)$ and $\mathrm{BP}\left(M_{\mathrm{BH}}=5.4 \pm 0.3 M_{\odot}\right)$. Since, for a given donor mass and orbital period, the MT rate tends to increase with decreasing BH mass, the small transient strip in the Hertzsprung gap may disappear when the more recent $\mathrm{BH}$ mass estimates are adopted.

$\mathrm{BP}$, finally, found that, using their estimates for the system parameters, both case A and early case B MT sequences are able to reproduce the observed system properties, but that case A sequences generally provided more satisfactory fits. Their example sequence 
with initial parameters $M_{\mathrm{BH}}=4.1 M_{\odot}, M_{2}=2.5 M_{\odot}$, and $P_{\text {orb }}=0.8$ days is close to our sequence BP35 (see Table 3), although the latter has a somewhat higher initial donor mass, possibly due to differences in the adopted convective overshooting.

A crucial element emerging from the MT calculations is the age of the donor star when all observational constraints are satisfied (i.e. the donor's current age). By using this age as an estimate for the time expired since the $\mathrm{BH}$ formation, we determined the possible birth sites of the $\mathrm{BH}$ by following the motion of the system in the Galaxy backwards in time. It follows that GRO J1655-40 was born between 3 and $7 \mathrm{kpc}$ from the Galactic center, and within $200 \mathrm{pc}$ from the Galactic plane. The post-SN peculiar velocity at the birth site can be anywhere between $\simeq 45$ and $\simeq 115 \mathrm{~km} \mathrm{~s}^{-1}$. We use this post-SN peculiar velocity to constrain the amount of mass lost in the SN explosion and the kick velocity that may have been imparted to the $\mathrm{BH}$. This is a major difference with previous investigations on the kinematics of GRO J1655-40 which used the present-day radial velocity (after correcting for the motion of the Sun and differential Galactic rotation, $V_{r}=-114 \pm 19 \mathrm{~km} \mathrm{~s}^{-1}$; Brandt et al. 1995) as a lower limit to the binary's center-of-mass velocity (see Brandt et al. 1995, Nelemans et al. 1999, Fryer \& Kalogera 2001).

The MT sequences also yield the age of the donor at the onset of RLO. Knowledge of this age allows us to link the post-SN orbital parameters to those at the onset of RLO by numerically integrating the system of differential equations governing the orbital evolution due to tides and gravitational radiation. Post-SN orbital semi-major axes and eccentricities compatible with successful MT sequences range from $A_{\text {postSN }}=5 R_{\odot}$ to $A_{\text {postSN }}=15 R_{\odot}$ and from $e_{\mathrm{postSN}}=0$ to $e_{\mathrm{postSN}}=0.35$.

The solution of the equations describing the conservation of orbital energy and angular momentum during compact object formation allows us to derive constraints on the pre-SN orbital separation, on the mass of the BH's helium star progenitor, and on the magnitude of the kick velocity that may have been imparted to the BH at birth. Despite the large number of successful MT sequences found, the progenitor constraints turn out to be fairly robust to the initial parameters of the sequences at the onset of RLO (see Fig. 7). Combining the constraints obtained for all successful MT sequences for both conservative and non-conservative MT, yields $M_{\mathrm{He}} \simeq 5.5-11.0 M_{\odot}$ and $V_{k} \simeq 30-160 \mathrm{~km} \mathrm{~s}^{-1}$ for GBO system parameters and $M_{\mathrm{He}} \simeq 3.5-9.0 M_{\odot}$ and $V_{k} \simeq 0-210 \mathrm{~km} \mathrm{~s}^{-1}$ for BP system parameters (see Fig. 8). It is clear that symmetric SN explosions lie at the edge of our solution ranges and that $\mathrm{BH}$ kicks of at least a few tens of $\mathrm{km} \mathrm{s}^{-1}$ are favored by the majority of the successful MT sequences (see Tables 2-3).

Hence, although some of the constraints associated with individual MT sequences require a kick to be imparted to the $\mathrm{BH}$ at birth (see Figs. 6-9 and Tables 2-3), without any addi- 
tional information, the still remaining uncertainties in the present-day system parameters at this stage do not allow us to exclude that the BH in GRO J1655-40 may have been formed through a symmetric SN explosion. In the final step of our investigation, we therefore model the core collapse using a 1D Lagrangian hydrodynamics code. From the energetics of the SN explosion, it follows that the mass of the BH's helium star progenitor must be larger than $8 M_{\odot}$. In addition, if the observed overabundances of heavy elements in the BH's companion are to be attributed to pollution from SN ejecta, the nucleosynthesis yields imply a maximum helium-star mass of $10 M_{\odot}$. Combining this with the constraints derived from the MT sequences, orbital dynamics, and motion in the Galaxy gives $30 \mathrm{~km} \mathrm{~s}^{-1} \lesssim V_{k} \lesssim 150 \mathrm{~km} \mathrm{~s}^{-1}$ in the case of GBO parameters and $40 \mathrm{~km} \mathrm{~s}^{-1} \lesssim V_{k} \lesssim 140 \mathrm{~km} \mathrm{~s}^{-1}$ in the case of BP parameters.

Our final set of constraints on the progenitor are much different than those obtained by Podsiadlowski et al. (2002b) who predict the likely progenitor to lie between $10-16 M_{\odot}$. But these differences are not due to different estimates of a given constraint, but the application of different constraints. Podsiadlowski et al. (2002b) focus their attention on the enrichment of the companion. As with our enrichment constraint (constraint III from §9), they find that more massive stars are better suited to explain the nucleosynthetic enrichment in the companion. Our constraint is slightly weaker, based on the simulations of Hungerford et al. (2003, 2004) which suggest that asymmetries can lead to very extended mixing. We have an additional limit based on both our mass-loss and supernova explosion model constraints (I and II from $\S 9$ ) that push for lower-mass progenitors. Since Podsiadlowski et al. (2002b) don't consider these constraints, they do not have this upper limit in their paper.

The question of whether or not a natal kick was imparted to the BH in GRO J165540 was addressed previously by Brandt et al. (1995), Nelemans et al. (1999), and Fryer \& Kalogera (2001). All three of these investigations used a lower limit on the present-day centerof-mass velocity given by the current radial velocity $\left(V_{r}=-114 \pm 19 \mathrm{~km} \mathrm{~s}^{-1}\right)$ instead of the actual post-SN peculiar velocity to constrain the mass loss and kick magnitude associated with the BH's formation. As illustrated in Fig. 4, this neglects changes in the system's center-of-mass velocity resulting from its acceleration in the Galactic potential. We here find that, even though following the Galactic motion of the system backward in time yields post-SN peculiar velocities all the way down to $\simeq 45 \mathrm{~km} \mathrm{~s}^{-1}$, the additional constraints on the binary properties make the possibility of a symmetric SN explosion only marginally acceptable solutions.

Jonker \& Nelemans (2004) recently reconsidered the vertical distribution of low-mass XRBs with respect to the Galactic plane and found no significant difference between NS and BH systems, suggesting that BHs may be subjected to natal kicks as well. The authors furthermore noted that the distances to Galactic soft X-ray transients may be systematically 
underestimated. If this is truly so, revised distance estimates may have important consequences for the determination of quiescent X-ray luminosities, peak outburst luminosities, BH masses, and Galactic distribution of BH XRBs. Clearly, if future measurements significantly revise any of the observational constraints, the analysis presented in this paper should be repeated to account for the most up-to-date knowledge of GRO J1655-40's current system properties.

In subsequent analyses, we intend to apply the procedure outlined above to the soft Xray transient XTE 1118+480, the high-mass XRBs Cyg X-1, LS 5039, LSI +61³03, Vela X-1, 4U1700-37, and the NS low-mass XRB Sco X-1. By examining both NS and BH systems and both RLO and stellar wind induced MT, we hope to unravel the systematic dependencies between the masses of newly formed compact objects and their immediate pre-SN progenitors, the mass lost at core collapse, and the possible kick velocity magnitude imparted to the compact object at birth. We expect that such constraints will help us understand better the physical origin of asymmetries in the collapse of massive stars.

We are indebted to Laura Blecha for sharing the code used to follow the motion of GRO J1655-40 in the Galactic potential, and to Jon Miller, Jerome Orosz, Jeffrey McClintock, Klaus Schenker, and Gijs Nelemans for useful and stimulating discussions. This work is supported by a David and Lucile Packard Foundation Fellowship in Science and Engineering grant and made extensive use of NASA's Astrophysics Data System Bibliographic Services.

\section{REFERENCES}

Ankay, A., Kaper, L., de Bruijne, J.H.J., Dewi, J., Hoogerwerf, R., \& Savonije, G.J. 2001, A\&A, 370, 170

Bailyn, C.D., et al. 1995a, Nature, 374, 701

Bailyn, C.D., Orosz, J.A., McClintock, J.E., \& Remillard, R.A. 1995b, Nature, 378, 157

Beer, M. E. \& Podsiadlowski, Ph. 2002, MNRAS 331, 351 (BP)

Belczynski, K., Kalogera, V., Taam, R.E., Rasio, F.A., Zezas, A., Bulik, T., \& Maccarone, T. 2004, in preparation

Benz, W., Hills, J.G., \& Thielemann, F.-K. 1989, ApJ, 342, 986

Blaauw, A. 1961, Bulletin of the Astronomical Institute of the Netherlands, 15, 265 
Blinnikov, S.I., Dunina-Barkovskaya, N.V., \& Nadyozhin, D.K. 1996, ApJS, 106,171

Boersma, J. 1961, Bulletin of the Astronomical Institute of the Netherlands, 15, 291

Brandt, N. \& Podsiadlowski, Ph. 1995, MNRAS, 274, 461

Brandt, W.N., Podsiadlowski, P., \& Sigurdsson, S. 1995, MNRAS, 277, L35

Carlberg, R.G. \& Innanen, K.A. 1987, AJ, 94, 666

Clark, G.W. 1975, ApJ, 199, L143

de Kool, M., van den Heuvel, E.P.J., \& Pylyser, E. 1987, A\&A, 183, 47

Dewi, J.D.M. \& van den Heuvel, E.P.J. 2004, MNRAS, 349, 169

Dubus, G., Lasota, J., Hameury, J., \& Charles, P. 1999, MNRAS, 303, 139

Eggleton, P.P. 1983, ApJ, 268, 368

Eggleton, P.P. \& Verbunt, F. 1986, MNRAS, 220, 13P

Ergma, E. \& van den Heuvel, E.P.J. 1998, A\&A, 331, L29

Flannery, B.P. \& van den Heuvel, E.P.J. 1975, A\&A, 39, 61

Fryer, C. 1999, ApJ, 522, 413

Fryer, C. \& Kalogera, V. 1997, ApJ, 489, 244

Fryer, C. \& Kalogera, V. 2001, ApJ, 554, 548

Fryer, C.L., Benz, W., Herant, M., \& Colgate, S.A. 1999, ApJ, 516, 892

Greene, J., Bailyn, C.D., \& Orosz, J.A. 2001, ApJ, 554, 1290 (GBO)

Gualandris, A., Colpi, M., Portegies Zwart, S., \& Possenti, A. 2005, ApJ, 618, 845

Heger, A., Fryer, C.L., Woosley, S.E., Langer, N., \& Hartmann, D.H. 2003, ApJ, 591, 288

Herant, M., Benz, W., Hix, W.R., Fryer, C.L., \& Colgate, S.A. 1994, ApJ, 435, 339

Hills, J.G. 1976, MNRAS, 175, 1P

Hills, J.G. 1983, ApJ, 267, 322

Hjellming, R.M. \& Rupen, M.P. 1995, Nature, 375, 464 
Humphreys, R.M. \& Larsen, J.A. 1995, AJ, 110, 2183

Hungerford, A.L., Fryer, C.L., \& Warren, M. S. 2003, ApJ, 594, 390

Hungerford, A.L., Fryer, C.L., Rockefeller, G., \& Timmes, F. X. 2004, in preparation

Hurley, J.R., Pols, O.R., \& Tout, C.A. 2000, MNRAS, 315, 543

Hurley, J.R., Tout, C.A., \& Pols, O.R. 2002, MNRAS, 329, 897

Hut, P. 1981, A\&A, 99, 126

Israelian, G., Rebolo, R., Basri, G., Casares, J., \& Martín, E.L. 1999, Nature, 401, 142

Ivanova, N., Belczynski, K., Kalogera, V., Rasio, F.A., \& Taam, R.E. 2003, ApJ, 592, 475

Jonker, P.G. \& Nelemans, G. 2004, MNRAS, 354, 355

Junker, W. \& Schäfer, G. 1992, MNRAS, 254, 146

Kalogera, V. 1996, ApJ, 471, 352

Kalogera, V. 1999, ApJ, 521, 723

Kalogera, V. 2000, ApJ, 541, 319

Kalogera, V. \& Lorimer, D.R. 2000, ApJ, 530, 890

Kalogera, V., Henninger, M., Ivanova, N., \& King, A.R. 2004, ApJ, 603, L41

King, A.R., Kolb, U., \& Burderi, L. 1996, ApJ, 464, L127

King, A.R., Kolb, U., \& Szuszkiewicz, E. 1997, ApJ, 488, 89

Kolb, U., King, A.R., Ritter, H., \& Frank, J. 1997, ApJ, 485, L33

Kolb, U. 1998, MNRAS, 297, 419

Kuijken, K. \& Gilmore, G. 1989, MNRAS, 239, 571

Lattimer, J.M., \& Swesty, F.D. 1991, Nucl. Phys. A., 535, 331

McClintock, J.E. \& Remillard, R.A. 2005, in Compact Stellar X-ray Sources, Eds. W.H.G. Lewin \& M. van der Klis, (Cambridge: Cambridge University Press), astro$\mathrm{ph} / 0306213$

McKay, D. \& Kesteven, M. 1994, IAU Circ., 6062, 2 
McKee, C.F. \& Hollenbach, D.J. 1980, ARA\&A, 18, 219

Mirabel, I.F., Dhawan, V., Mignani, R.P., Rodrigues, I., \& Guglielmetti, F. 2001, Nature, 413, 139

Mirabel, I.F., Mignani, R., Rodrigues, I., Combi, J.A., Rodríguez, L.F., \& Guglielmetti, F. 2002, A\&A, 395, 595

Mirabel, I.F. \& Rodrigues, I. 2003a, Science, 300, 1119

Mirabel, I.F. \& Rodrigues, I. 2003b, A\&A, 398, L25

Mirabel, I.F., Rodrigues, I., \& Liu, Q.Z. 2004, A\&A, 422, L29

Nelemans, G., Tauris, T.M., \& van den Heuvel, E.P.J. 1999, A\&A, 352, L87

Orosz, J.A. 2004, http://mintaka.sdsu.edu/faculty/orosz/web/

Orosz, J.A. \& Bailyn, C.D. 1997, ApJ, 477, 876

Phillips, S.N., Shahbaz, T., \& Podsiadlowski, P. 1999, MNRAS, 304, 839

Podsiadlowski, P., Rappaport, S., \& Pfahl, E.D. 2002a, ApJ, 565, 1107

Podsiadlowski, P., Nomoto, K., Maeda, K., Nakamura, T., Mazzali, P., \& Schmidt, B. 2002b, ApJ, 567, 491

Podsiadlowski, P., Rappaport, S., \& Han, Z. 2003, MNRAS, 341, 385

Portegies Zwart, S.F., Verbunt, F., \& Ergma, E. 1997, A\&A, 321, 207

Rauscher, T., Heger, A., Hoffman, R.D., \& Woosley, S.E. 2003, Nucl. Phys. A, 718, 463

Regős, E., Tout, C.A., \& Wickramasinghe, D. 1998, ApJ, 509, 362

Reid, M.J. 1993, ARA\&A, 31, 345

Ribó, M., Paredes, J.M., Romero, G.E., Benaglia, P., Martí, J., Fors, O., \& García-Sánchez, J. 2002, A\&A, 384, 954

Romani, R.W. 1996, IAU Symp. 165: Compact Stars in Binaries, 165, 93

Shahbaz, T. 2003, MNRAS, 339, 1031

Shahbaz, T., van der Hooft, F., Casares, J., Charles, P.A., \& van Paradijs, J. 1999, MNRAS, 306, 89 
Soria, R., Wickramasinghe, D.T., Hunstead, R.W., \& Wu, K. 1998, ApJ, 495, L95

Timmes, F. X. 1999, ApJS, 124, 241

Tingay, S.J., et al. 1995, Nature, 374, 141

van der Hooft, F., Heemskerk, M.H.M., Alberts, F., \& van Paradijs, J. 1998, A\&A, 329, 538

Wellstein, S., \& Langer, N. 1999, A\&A, 350, 148

Willems, B. \& Kalogera, V. 2004, ApJ, 603, L101

Willems, B., Kalogera, V., \& Henninger, M. 2004, ApJ, in press

Witte, M.G. \& Savonije, G.J. 1999, A\&A, 350, 129

Zahn, J.-P. 1975, A\&A, 41, 329

Zahn, J.-P. 1977, A\&A, 57, 383

Zahn, J.-P. 1978, A\&A, 67, 162

Zhang, S.N., Wilson, C.A., Harmon, B.A., Fishman, G.J., Wilson, R.B., Paciesas, W.S., Scott, M., \& Rubin, B.C. 1994, IAU Circ., 6046, 1 